\title{
Continuity properties of transport coefficients in simple maps
}

\author{
Gerhard Keller $^{1}$, Phil J. Howard ${ }^{2}$, Rainer Klages ${ }^{2}$ \\ ${ }^{1}$ Mathematisches Institut, Universität Erlangen-Nürnberg

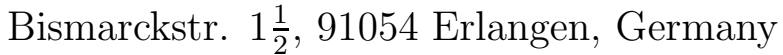 \\ ${ }^{2}$ School of Mathematical Sciences, Queen Mary, University of London, \\ Mile End Road, London E1 4NS, UK
}

April 25, 2022

\begin{abstract}
We consider families of dynamics that can be described in terms of Perron-Frobenius operators with exponential mixing properties. For piecewise $C^{2}$ expanding interval maps we rigorously prove continuity properties of the drift $J(\lambda)$ and of the diffusion coefficient $D(\lambda)$ under parameter variation. Our main result is that $D(\lambda)$ has a modulus of continuity of order $\left.\mathcal{O}(|\delta \lambda| \cdot|\log | \delta \lambda \mid)^{2}\right)$, i.e. $D(\lambda)$ is Lipschitz continuous up to quadratic logarithmic corrections. For a special class of piecewise linear maps we provide more precise estimates at specific parameter values. Our analytical findings are verified numerically for the latter class of maps by using exact formulas for the transport coefficients. We numerically observe strong local variations of all continuity properties.
\end{abstract}

\section{Introduction}

In simple deterministic dynamical systems physical quantities like transport coefficients can be fractal functions of control parameters. This finding was first reported for a one-dimensional piecewise linear map lifted periodically onto the whole real line, for which the diffusion coefficient was computed by using Markov partitions and topological transition matrices [26, 27, 29]. A generalization of this result was obtained for a map with both drift and diffusion by deriving exact analytical solutions for the transport coefficients [15,9]. Further maps modeling chemical reaction-diffusion [14] and anomalous diffusion [33] yielded also fractal transport coefficients. Recent work aimed at physically more realistic models like (Hamiltonian) particle billiards, for which computer simulations yielded transport coefficients that are non-monotonic under parameter variation 31. Ref. 32] contains a summary of this line of research.

These results asked for a more detailed characterization of the "fractality" of transport coefficients. A first attempt in this direction was reported by Klages and Klauß [30, who used standard techniques from the theory of fractal dimensions for characterizing the drift and diffusion coefficients of the map studied in [15]. They numerically computed a non-integer box counting dimension for these curves which varied with the parameter interval, leading to the notion of a "fractal fractal dimension". These results were questioned by Koza 35, who computed the oscillation of these graphs at specific Markov partition parameter values. His work suggested a dimensionality of one by conjecturing that there exist non-trivial logarithmic corrections to the usual power law behaviour in the oscillation.

This research reveals the need to study the parameter dependence of transport coefficients in a rigorous mathematical setting, which can be formulated as follows: Given a parametrized family of chaotic dynamical systems $T_{\lambda}: I \rightarrow I$ on an interval $I$ with unique invariant physical measures $\mu_{\lambda}$ together with a family of sufficiently regular observables $\psi_{\lambda}: I \rightarrow \mathbb{R}$ one has, under 
suitable mixing assumptions on the systems $\left(T_{\lambda}, \mu_{\lambda}\right)$, a law of large numbers and a central limit theorem for the partial sum processes $S_{\lambda, n}(x)=\sum_{k=0}^{n-1} \psi_{\lambda}\left(T_{\lambda}^{k} x\right)$, namely

$$
\lim _{n \rightarrow \infty} n^{-1} S_{\lambda, n}=J(\lambda):=\int_{I} \psi_{\lambda}(x) d \mu_{\lambda}(x) \text { for } \mu_{t} \text {-a.e. } x
$$

and

$$
\mathcal{L}\left(n^{-\frac{1}{2}} S_{\lambda, n}\right) \Rightarrow \mathcal{N}(0,2 D(\lambda))
$$

where $D(\lambda):=\lim _{n \rightarrow \infty} \frac{1}{2 n} \int_{I}\left(\sum_{k=0}^{n-1}\left(\psi_{\lambda}\left(T_{\lambda}^{k} x\right)-J(\lambda)\right)\right)^{2} d \mu_{\lambda}(x)$. For suitable choices of the observables $\psi_{\lambda}$, the process $S_{\lambda, n}$ is just the deterministic random walk generated by a lift of the map $T_{\lambda}$ to the real axis, and $J(\lambda)$ and $D(\lambda)$ are the drift and diffusion coefficient of this random walk respectively.

There are a few rigorous results in the literature describing the dependence of $\mu_{\lambda}$ and of quantities like $J(\lambda)$ for various classes of systems. Without going into the details they can be summarized as follows: If the maps $T_{\lambda}$ and the observables $\psi_{\lambda}$ depend smoothly on $\lambda$ and if the topological conjugacy class of $T_{\lambda}$ is not changed when $\lambda$ is varied, then $\mu_{\lambda}$ (and hence $J(\lambda)$ ) depends differentiably on $\lambda[4,8,10,19,20,39,40$. If the topological class changes, quantities like $J(\lambda)$ may behave less regular and have a modulus of continuity not better than $|\delta \lambda \cdot \log | \delta \lambda \|$, even for very simple maps $T_{\lambda}$ like symmetric tent maps [3]. On the other hand, this modulus of continuity is the rule for systems whose Perron-Frobenius operator (acting on a suitable space of "regular" densities) has a spectral gap [22,24].

The goal of this paper is to explicitly relate these mathematical results to transport coefficients. We do so by rigorously proving continuity properties of $J(\lambda)$ and $D(\lambda)$ under parameter variation for certain classes of deterministic maps. In Section 2 we give a general estimate for families of dynamics (deterministic or not), which can be described in terms of PerronFrobenius operators with exponential mixing properties. The applicability of these general results to piecewise $C^{2}$ expanding interval maps and in particular to the class of piecewise linear maps discussed in [26, 27, 29, 15, 32 is checked in Section 3. The main result is that $D(\lambda)$ has a modulus of continuity of order $\left.\mathcal{O}(|\delta \lambda| \cdot|\log | \delta \lambda \mid)^{2}\right)$, i.e. $D(\lambda)$ is Lipschitz continuous up to quadratic logarithmic corrections. In Section 4 we summarize the general results for transport coefficients in the special case of piecewise linear maps and provide more precise estimates for special parameters. Our analytical findings are verified by numerical computations in Section 5 . for which we use exact analytical formulas of the transport coefficients [15]. Particularly, we numerically analyze local variations of these properties. Our work corrects and amends previous results reported in 30,35 .

\section{The general setting}

Let $I$ be a compact interval, $m$ normalized Lebesgue measure on $I, L_{m}^{1}$ the space of Lebesgueintegrable functions from $I$ to $\mathbb{R}$, and $B V \subset L_{m}^{1}$ the space of $L_{m}^{1}$-equivalence classes of functions of bounded variation. We use the following simplified notation for the two corresponding norms:

$$
|f|_{1}:=\int|f| d m, \quad\|f\|:=\operatorname{Var}(f)
$$

where

$$
\operatorname{Var}(f):=\sup \left\{\int f \varphi^{\prime} d m: \varphi \in C^{1}(\mathbb{R}, \mathbb{R}),|\varphi|_{\infty} \leq 1\right\}
$$

is the variation of $f$ as a function from $\mathbb{R} \rightarrow \mathbb{R}$ (i.e. extended by $f \equiv 0$ on $\mathbb{R} \backslash I$ ). If $f$ is differentiable as a function from $\mathbb{R} \rightarrow \mathbb{R}$ integration by parts shows easily that $\operatorname{Var}(f)=$ $\int\left|f^{\prime}\right| d m$. Var is obviously a semi-norm, and as $|f|_{1} \leq|f|_{\infty} \leq \frac{1}{2} \operatorname{Var}(f)$, it is actually a norm. This and more details on functions of bounded variation can be found in [25, section 2.3]. The monograph [2] is a comprehensive reference for most of the background material needed in this section. 
We consider a family $\mathcal{T}$ of nonsingular maps $T: I \rightarrow I$. Nonsingular means that the Perron-Frobenius operator $P_{T}: L_{m}^{1} \rightarrow L_{m}^{1}$ is well defined, i.e.

$$
\int P_{T} f \cdot g d m=\int f \cdot g \circ T d m \quad\left(f \in L_{m}^{1}, g \in L_{m}^{\infty}\right) .
$$

By definition, $\left|P_{T}\right|_{1}=1$ for all $T \in \mathcal{T}$, and we assume

$$
\text { Hypothesis } 1 \quad C_{1}:=\sup \left\{\left\|P_{T}^{n}\right\|: T \in \mathcal{T}, n \in \mathbb{N}\right\}<\infty .
$$

Our main assumption is that the maps in $\mathcal{T}$ are uniformly exponentially mixing in the following sense:

Hypothesis 2 Each $T \in \mathcal{T}$ has a unique invariant probability density $h_{T} \in B V$ (so $P_{T} h_{T}=$ $\left.h_{T}\right)$, and there are constants $\gamma \in(0,1)$ and $C_{2}>0$ such that, for all $T \in \mathcal{T}$,

$$
\left|P_{T}^{n} f\right|_{1} \leq C_{2} \gamma^{n} \operatorname{Var}(f) \quad \text { for all } f \in B V \text { with } \int f d m=0 \text { and for all } n \in \mathbb{N} .
$$

Observe the following consequences of Hypothesis 1 and 2 ,

$$
\left|P_{T}^{n} f-h_{T}\right|_{1} \leq C_{2} \gamma^{n}\left(\operatorname{Var}(f)+2 C_{1}\right) \quad \text { for all probability densities } f \in B V
$$

and

$$
\operatorname{Var}\left(h_{T}\right) \leq 2 C_{1} \quad(T \in \mathcal{T}) .
$$

Indeed, $\left|P_{T}^{n} f-h_{T}\right|_{1}=\left|P_{T}^{n}\left(f-h_{T}\right)\right|_{1} \leq C_{2} \gamma^{n}\left(\operatorname{Var}(f)+\operatorname{Var}\left(h_{T}\right)\right) \rightarrow 0$ as $n \rightarrow \infty$ by Hypothesis 2 for each probability density $f \in B V$, and $\operatorname{Var}\left(P_{T}^{n} 1\right) \leq C_{1} \operatorname{Var}(1)=2 C_{1}$ by Hypothesis 1 . Hence (6) follows from the definition (2) of $\operatorname{Var}($.$) , and then (5) is an immediate consequence.$

Since it is our goal to investigate the dependence of various dynamical quantities as functions of $T \in \mathcal{T}$, we need to introduce a distance on $\mathcal{T}$. At this stage the following one, which was already considered in 22], is most apropriate. It measures the distance between two maps $T_{1}$ and $T_{2}$ from $\mathcal{T}$ in terms of a suitable norm of $P_{T_{1}}-P_{T_{2}}$ :

$$
\left\|P_{T_{1}}-P_{T_{2}} \mid\right\|:=\sup \left\{\left|P_{T_{1}} f-P_{T_{2}} f\right|_{1}: f \in B V,\|f\| \leq 1\right\} .
$$

This distance can be controlled in terms of a more "hands-on" distance between the graphs of the maps:

$$
\begin{aligned}
& d\left(T_{1}, T_{2}\right):=\inf \left\{\epsilon>0: \exists I_{\epsilon} \subseteq I \text { and } \exists \text { a diffeomorphism } \sigma: I \rightarrow I\right. \text { s.th. } \\
& m\left(I \backslash I_{\epsilon}\right)<\epsilon,\left.T_{1}\right|_{I_{\epsilon}}=\left.T_{2} \circ \sigma\right|_{I_{\epsilon}}, \text { and } \\
&\left.\forall x \in I_{\epsilon}:|\sigma(x)-x|<\epsilon,\left|1 / \sigma^{\prime}(x)-1\right|<\epsilon\right\} .
\end{aligned}
$$

Namely (see [22, Lemma 13]),

$$
\left\|P_{T_{1}}-P_{T_{2}}\right\| \leq 12 \cdot d\left(T_{1}, T_{2}\right) .
$$

Now, as a warm-up exercise, we can prove the following estimate: for $k \geq 0$ let

$$
\ell_{k}:(0, \infty) \rightarrow(0, \infty), \quad \ell_{k}(u):=u \cdot(1+|\log u|)^{k} .
$$

Lemma 1 There exist constants $K_{1}^{\prime}, K_{1}>0$ such that

$$
\left|h_{T_{1}}-h_{T_{2}}\right|_{1} \leq K_{1}^{\prime} \cdot \ell_{1}\left(\left\|\mid P_{T_{1}}-P_{T_{2}}\right\|\right) \leq K_{1} \cdot \ell_{1}\left(d\left(T_{1}, T_{2}\right)\right) \quad\left(T_{1}, T_{2} \in \mathcal{T}\right)
$$


Proof: Let $\tilde{\eta}:=\left\|P_{T_{1}}-P_{T_{2}}\right\|$, assume without loss of generality that $\tilde{\eta}<1$, and fix $N \in \mathbb{N}$. For $f \in B V$,

$$
\begin{aligned}
\left|P_{T_{1}}^{N} f-P_{T_{2}}^{N} f\right|_{1} & \leq \sum_{k=0}^{N-1}\left|P_{T_{1}}^{N-k-1}\left(P_{T_{1}}-P_{T_{2}}\right) P_{T_{2}}^{k} f\right|_{1} \leq \sum_{k=0}^{N-1}\left|\left(P_{T_{1}}-P_{T_{2}}\right) P_{T_{2}}^{k} f\right|_{1} \\
& \leq \sum_{k=0}^{N-1} \mid\left\|P_{T_{1}}-P_{T_{2}}\right\|\left\|P_{T_{2}}^{k} f\right\| \leq \sum_{k=0}^{N-1} \tilde{\eta} C_{1}\|f\| \leq C_{1} N \tilde{\eta}\|f\|
\end{aligned}
$$

where we used Hypothesis 1. Hence,

$$
\begin{aligned}
\left|h_{T_{1}}-h_{T_{2}}\right|_{1} & \leq\left|P_{T_{1}}^{N} 1-P_{T_{2}}^{N} 1\right|_{1}+\left|P_{T_{1}}^{N}\left(1-h_{T_{1}}\right)\right|_{1}+\left|P_{T_{2}}^{N}\left(1-h_{T_{2}}\right)\right|_{1} \\
& \leq 2 C_{1} N \tilde{\eta}+2 \cdot C_{2} \gamma^{N}\left(2+2 C_{1}\right)
\end{aligned}
$$

where we used (4) and (6). With $N=\left\lceil\frac{\log \tilde{\eta}}{\log \gamma}\right\rceil$, this is (11).

q.e.d.

Remark 1 Even if $\mathcal{T}$ is a family of piecewise linear maps and if $T_{1}$ has the Markov property, this estimate can generally not be improved. Examples for this fact within the family of symmetric mixing tent maps are provided in [3, 37].

Suppose now that to each $T \in \mathcal{T}$ there is associated an "observable" $\psi_{T}: I \rightarrow \mathbb{R}$. We make the following assumptions:

Hypothesis $3 \quad C_{3}:=\sup \left\{\operatorname{Var}\left(\psi_{T}\right): T \in \mathcal{T}\right\}<\infty$

Hypothesis 4 There is $C_{4}>0$ such that $\left|\psi_{T_{1}}-\psi_{T_{2}}\right|_{1} \leq C_{4} d\left(T_{1}, T_{2}\right)$ for all $T_{1}, T_{2} \in \mathcal{T}$.

Denote

$$
J(T):=\int_{I} \psi_{T} h_{T} d m .
$$

Then we have immediately from ([6) and Lemma 1

Corollary 1 There is some $K_{2}>0$ such that, for all $T_{1}, T_{2} \in \mathcal{T}$,

$$
\left|J\left(T_{1}\right)-J\left(T_{2}\right)\right| \leq K_{2} \cdot \ell_{1}\left(d\left(T_{1}, T_{2}\right)\right)
$$

$J(T)$ is the "drift" of the partial sum process

$$
S_{T, n}:=\sum_{k=0}^{n-1} \psi_{T} \circ T^{k}=n J(T)+\sum_{k=0}^{n-1} \hat{\psi}_{T} \circ T^{k}
$$

under the invariant measure $h_{T} m$, where $\hat{\psi}_{T}=\psi_{T}-J(T)$. Observe that

$$
\operatorname{Var}\left(\hat{\psi}_{T}\right) \leq 2 C_{3}, \quad\left|\psi_{T_{1}}-\psi_{T_{2}}\right| \leq 2 C_{4} d\left(T_{1}, T_{2}\right) \quad \text { for all } T, T_{1}, T_{2} \in \mathcal{T} .
$$

In view of Hypothesis 2 we can also define the "diffusion coefficient"' 1 of this process:

$$
\begin{aligned}
D(T) & :=\lim _{n \rightarrow \infty} \frac{1}{2 n} \int\left(\sum_{k=0}^{n-1} \hat{\psi}_{T} \circ T^{k}\right)^{2} h_{T} d m \\
& =\frac{1}{2} \int \hat{\psi}_{T}^{2} h_{T} d m+\sum_{n=1}^{\infty} \int \hat{\psi}_{T} \cdot \hat{\psi}_{T} \circ T^{n} h_{T} d m \\
& =\frac{1}{2} \int \hat{\psi}_{T}^{2} h_{T} d m+\sum_{n=1}^{\infty} \int P_{T}^{n}\left(\hat{\psi}_{T} h_{T}\right) \hat{\psi}_{T} d m
\end{aligned}
$$

${ }^{1}$ This is the convention in the physics literature. In the mathematics literature one would rather call $2 D(T)$ the diffusion coefficient. 
Even more, we have the central limit theorem

$$
\mathcal{L}\left(n^{-\frac{1}{2}}\left(S_{T, n}-n J(T)\right) \Rightarrow \mathcal{N}(0,2 D(T)) \text { as } n \rightarrow \infty,\right.
$$

see e.g. [21, 18, 38]. Among physicists (16) is known as the Taylor-Green-Kubo formula for diffusion [32. For the dependence of $D(T)$ on $T$ we prove:

Proposition 1 There is some $K_{3}>0$ such that, for all $T_{1}, T_{2} \in \mathcal{T}$,

$$
\left|D\left(T_{1}\right)-D\left(T_{2}\right)\right| \leq K_{3} \cdot \ell_{2}\left(d\left(T_{1}, T_{2}\right)\right)
$$

Proof: Observe first that, for all $\psi, h \in B V$,

$$
\operatorname{Var}(\psi h) \leq \operatorname{Var}(\psi)|h|_{\infty}+\operatorname{Var}(h)|\psi|_{\infty} \leq \operatorname{Var}(\psi) \operatorname{Var}(h)
$$

Indeed, for differentiable $u$ we have $\operatorname{Var}(u)=\int\left|u^{\prime}\right| d m$, so for differentiable $h$ and $\psi$ (19) follows from the product rule of diferentiation. General $h$ and $\psi$ are then approximated using mollifiers. It follows that, in view of (4),

$$
\begin{aligned}
\left|\int P_{T}^{n}\left(\hat{\psi}_{T} h_{T}\right) \hat{\psi}_{T} d m\right| & \leq\left|P_{T}^{n}\left(\hat{\psi}_{T} h_{T}\right)\right|_{1} \cdot\left|\hat{\psi}_{T}\right|_{\infty} \leq C_{2} C_{3} \gamma^{n} \operatorname{Var}\left(\hat{\psi}_{T} h_{T}\right) \\
& \leq 4 C_{1} C_{2} C_{3}^{2} \gamma^{n}
\end{aligned}
$$

Let $\tilde{\eta}=\left\|P_{T_{1}}-P_{T_{2}}\right\|$ as before, denote $\eta:=d\left(T_{1}, T_{2}\right)$ (so that $\tilde{\eta} \leq 12 \eta$ ), and fix $N=\left\lceil\frac{\log \eta}{\log \gamma}\right\rceil$. For all $T_{1}, T_{2} \in \mathcal{T}$, eq. (20) implies

$$
2 \sum_{n=N}^{\infty}\left|\int P_{T_{1}}^{n}\left(\hat{\psi}_{T_{1}} h_{T_{1}}\right) \hat{\psi}_{T_{1}} d m-\int P_{T_{2}}^{n}\left(\hat{\psi}_{T_{2}} h_{T_{2}}\right) \hat{\psi}_{T_{2}} d m\right| \leq \frac{16 C_{1} C_{2} C_{3}^{2}}{1-\gamma} \eta .
$$

For $0 \leq n<N$ we use a different estimate. We decompose

$$
\int P_{T_{1}}^{n}\left(\hat{\psi}_{T_{1}} h_{T_{1}}\right) \hat{\psi}_{T_{1}} d m-\int P_{T_{2}}^{n}\left(\hat{\psi}_{T_{2}} h_{T_{2}}\right) \hat{\psi}_{T_{2}} d m=\Delta_{1}^{n}+\Delta_{2}^{n}+\Delta_{3}^{n}+\Delta_{4}^{n}
$$

where

$$
\begin{aligned}
\left|\Delta_{1}^{n}\right| & :=\left|\int P_{T_{1}}^{n}\left(\hat{\psi}_{T_{1}} h_{T_{1}}\right)\left(\hat{\psi}_{T_{1}}-\hat{\psi}_{T_{2}}\right) d m\right| \leq \frac{1}{2} \operatorname{Var}\left(P_{T_{1}}^{n}\left(\hat{\psi}_{T_{1}} h_{T_{1}}\right)\right)\left|\hat{\psi}_{T_{1}}-\hat{\psi}_{T_{2}}\right|_{1} \\
& \leq 4 C_{1}^{2} C_{3} C_{4} \eta
\end{aligned}
$$

and

$$
\begin{aligned}
\left|\Delta_{2}^{n}\right| & :=\left|\int\left(P_{T_{1}}^{n}-P_{T_{2}}^{n}\right)\left(\hat{\psi}_{T_{1}} h_{T_{1}}\right) \hat{\psi}_{T_{2}} d m\right| \leq||\left|P_{T_{1}}^{n}-P_{T_{2}}^{n} \|\right| \operatorname{Var}\left(\hat{\psi}_{T_{1}} h_{T_{1}}\right)\left|\hat{\psi}_{T_{2}}\right|_{\infty} \\
& \leq 4 C_{1} C_{3}^{2}\left\|\left|P_{T_{1}}^{n}-P_{T_{2}}^{n} \|\right| \leq 4\left(C_{1} C_{3}\right)^{2} n \tilde{\eta}\right.
\end{aligned}
$$

where the last inequality follows from eq. (12). Next,

$$
\begin{aligned}
\left|\Delta_{3}^{n}\right|: & =\left|\int P_{T_{2}}^{n}\left(\left(\hat{\psi}_{T_{1}}-\hat{\psi}_{T_{2}}\right) h_{T_{1}}\right) \hat{\psi}_{T_{2}} d m\right| \leq\left|\hat{\psi}_{T_{1}}-\hat{\psi}_{T_{2}}\right|_{1}\left|h_{T_{1}}\right|_{\infty}\left|\hat{\psi}_{T_{2}}\right|_{\infty} \\
& \leq 4 C_{1} C_{3} C_{4} \eta
\end{aligned}
$$

and

$$
\begin{aligned}
\left|\Delta_{4}^{n}\right|: & =\left|\int P_{T_{2}}^{n}\left(\hat{\psi}_{T_{2}}\left(h_{T_{1}}-h_{T_{2}}\right)\right) \hat{\psi}_{T_{2}} d m\right| \leq\left|h_{T_{1}}-h_{T_{2}}\right|_{1}\left|\hat{\psi}_{T_{2}}\right|_{\infty}\left|\hat{\psi}_{T_{2}}\right|_{\infty} \\
& \leq C_{3}^{2} K_{1} \ell_{1}(\tilde{\eta})
\end{aligned}
$$


¿From (23) - (26) we see that

$$
\left|\Delta_{1}^{n}\right|+\left|\Delta_{2}^{n}\right|+\left|\Delta_{3}^{n}\right|+\left|\Delta_{4}^{n}\right| \leq \tilde{K}\left(n \eta+\ell_{1}(\eta)\right)
$$

for some constant $\tilde{K}>0$. Hence, in view of (21) and the choice of $N$,

$$
\begin{aligned}
\left|D\left(T_{1}\right)-D\left(T_{2}\right)\right| & \leq \frac{4 C_{1} C_{2} C_{3}^{2}}{1-\gamma} \eta+\tilde{K}\left(\ell_{1}(\eta)+2 \sum_{n=1}^{N-1}\left(n \eta+\ell_{1}(\eta)\right)\right) \\
& \leq \frac{4 C_{1} C_{2} C_{3}^{2}}{1-\gamma} \eta+\tilde{K}\left(N^{2} \eta+(2 N-1) \ell_{1}(\eta)\right) \\
& \leq K_{3} \cdot \ell_{2}(\eta)
\end{aligned}
$$

for a suitable constant $K_{3}$.

q.e.d.

Remark 2 Quite often slightly stronger forms of Hypotheses 2 and 4 are satisfied, where the mixing assumption (4) is replaced by

$$
\operatorname{Var}\left(P_{T}^{n} f\right) \leq C_{2}^{\prime} \gamma^{n} \operatorname{Var}(f) \text { for all } f \in B V \text { with } \int f d m=0 \text { and } n \in \mathbb{N}
$$

and the assumption on the $T$-dependence of $\psi_{T}$ is strengthened to

$$
\operatorname{Var}\left(\psi_{T_{1}}-\psi_{T_{2}}\right) \leq C_{4}^{\prime} d\left(T_{1}, T_{2}\right) \quad \text { for all } T_{1}, T_{2} \in \mathcal{T}
$$

An inspection of the above estimates shows that $\left|\Delta_{1}^{n}\right| \leq 4 C_{1} C_{2}^{\prime} C_{3} C_{4} \gamma^{n} \eta$ and $\left|\Delta_{2}^{n}\right| \leq 4 C_{1} C_{2}^{\prime} C_{3}(1-$ $\gamma)^{-1} \eta$ if (29) is assumed. If additionally (30) is assumed, then $\left|\Delta_{3}^{n}\right|$ can be estimated as follows: Let $\alpha:=\int_{I}\left(\hat{\psi}_{T_{1}}-\hat{\psi}_{T_{2}}\right) h_{T_{1}} d m$. Then

$$
\begin{aligned}
\left|\Delta_{3}^{n}\right| & =\left|\int P_{T_{2}}^{n}\left(\left(\hat{\psi}_{T_{1}}-\hat{\psi}_{T_{2}}\right) h_{T_{1}}-\alpha h_{T_{2}}\right) \hat{\psi}_{T_{2}} d m\right| \leq C_{2} C_{3} \gamma^{n} \operatorname{Var}\left(\left(\hat{\psi}_{T_{1}}-\hat{\psi}_{T_{2}}\right) h_{T_{1}}-\alpha h_{T_{2}}\right) \\
& \leq C_{1} C_{2} C_{3} C_{4}^{\prime}(4+2) \gamma^{n} \eta .
\end{aligned}
$$

Hence, $\sum_{n=0}^{N-1}\left|\Delta_{1}^{n}\right|+\left|\Delta_{3}^{n}\right|=\mathcal{O}(\eta)$ uniformly in $N$ and $\sum_{n=0}^{N-1}\left|\Delta_{2}^{n}\right|=\mathcal{O}(N \eta)=\mathcal{O}\left(\ell_{1}(\eta)\right)$. But we see no way, in general, to bound the $\Delta_{4}^{n}$-terms in a similar way. However, for particular families of maps (which are all topologically conjugate), we will see in subsection 4.2 that $\Delta_{4}^{n}=0$ for all $n$ and that the estimate for $\left|\Delta_{2}^{n}\right|$ can be made more precise.

\section{Checking Hypothesis 1 and 2}

\subsection{General piecewise expanding maps}

In this subsection we show how the general Hypothesis 1 and 2 can be verified in the more particular setting when $\mathcal{T}$ is a parametrized family of piecewise twice continuously differentiable and expanding interval maps. So, from now on, we look at the following setting:

$\Lambda \subset \mathbb{R}^{d}$ is a compact parameter space, $\mathcal{T}=\left\{T_{\lambda}: \lambda \in \Lambda\right\}$, and

there is some $L>0$ such that $d\left(T_{\lambda_{1}}, T_{\lambda_{2}}\right) \leq L\left|\lambda_{1}-\lambda_{2}\right|$ for all $\lambda_{1}, \lambda_{2} \in \Lambda$.

We start with an abstract result which reduces Hypothesis 2 essentially to a uniform LasotaYorke type inequality.

Lemma 2 Assume (T1) and (T2). Then Hypothesis 1 and 2 are valid if the transformations $T \in \mathcal{T}$ are mixing and satisfy a uniform Lasota-Yorke type inequality: there are constants $C_{5}, C_{6}>0$ and $\alpha \in(0,1)$ such that

$$
\operatorname{Var}\left(P_{T}^{n} f\right) \leq C_{5} \alpha^{n} \operatorname{Var}(f)+C_{6}|f|_{1} \quad \text { for all } T \in \mathcal{T}, n \in \mathbb{N}, f \in B V .
$$


Proof: As $\left|P_{T}\right|_{1}=1$ and $|f|_{1} \leq \frac{1}{2}\|f\|$, it is straightforward to check that Hypothesis 1 holds with $C_{1}=C_{5}+\frac{1}{2} C_{6}$.

We turn to Hypothesis 2 Note first that, because of (T1) and (T2), it suffices to show that for each $\lambda \in \Lambda$ there are $\delta(\lambda)>0, C_{2}(\lambda)>0$ and $\gamma(\lambda) \in(0,1)$ such that (4) holds with these constants for all $T_{\lambda_{1}}$ with $\left|\lambda_{1}-\lambda\right|<\delta(\lambda)$. But this is guaranteed by Corollary 2 and Remark $1 \mathrm{c}$ in $[24$.

q.e.d.

Our next task is to give sufficient conditions for $(\overline{L Y})$. To this end we specialize further and assume from now on that our maps are piecewise expanding (PE) maps in the following sense:

For each $\lambda \in \Lambda$ there is a finite partition $\left(I_{\lambda}^{1}, \ldots, I_{\lambda}^{N_{\lambda}}\right)$ of $I$ into subintervals such that all $\left.T_{\lambda}\right|_{I_{\lambda}^{j}}$ are monotone, $C^{2}$, and $\kappa_{\lambda}:=\inf \left|T_{\lambda}^{\prime}\right|>2$.

Already in [36] it was proved that each individual (PE)-map (even if $1<\kappa_{\lambda} \leq 2$ ) satisfies (LY) with constants $C_{5}, C_{6}, \alpha$ depending on the map. For parametrized families of maps one can generally find uniform constants, but there are counterexamples where this is not possible [22,6, 7. Under the above assumption $\inf _{\lambda \in \Lambda} \kappa_{\lambda}>2$ one can, however, give simple sufficient conditions ensuring the uniform LY-inequality. The proof in [36] (see also [25, Proposition 2.1]) shows

$$
\operatorname{Var}\left(P_{T_{\lambda}} f\right) \leq \frac{2}{\kappa_{\lambda}} \operatorname{Var}(f)+\left(D_{\lambda}+E_{\lambda}\right)|f|_{1}
$$

where

$$
D_{\lambda}=\sup _{x}\left|\left(\frac{1}{T_{\lambda}^{\prime}(x)}\right)^{\prime}\right|, \quad E_{\lambda}=\frac{2}{\kappa_{\lambda} \min _{j}\left|I_{\lambda}^{j}\right|} .
$$

From this (LY) follows with $\alpha=\frac{2}{\kappa_{\lambda}}, C_{5}=1$, and $C_{6}=\sup _{\lambda} \frac{\kappa_{\lambda}}{\kappa_{\lambda}-2}\left(D_{\lambda}+E_{\lambda}\right)$ provided this supremum is finite.

In some cases of interest the $E_{\lambda}, \lambda \in \Lambda$, are not bounded because there are arbitrarily short monotonicity intervals. In such situations, ad hoc arguments are needed. We give an example in the next section.

\subsection{Piecewise linear modulo 1 maps}

We now look at a particular model dealt with in [12,13,16,17, from a mathematical perspective and in $\left[26,27,29,15,32\right.$ from a physics point of view. Let $I=\left[-\frac{1}{2}, \frac{1}{2}\right], \Lambda=\left[a_{0}, a_{1}\right] \times\left[-\frac{1}{2}, \frac{1}{2}\right]$ for some constants $2<a_{0}<a_{1}$, and for $\lambda=(a, b) \in \Lambda$ consider

$$
T_{\lambda}(x)=a x+b \bmod \left(\mathbb{Z}-\frac{1}{2}\right) .
$$

Hofbauer [16] showed that these maps have always a unique invariant probability density 2, but although these maps received further attention also in the mathematical literature [17,12],13, it is not so easy to draw Hypothesis 2 from these sources. Therefore we will take up a rather direct computation made in 23] to prove, without having to rely on the compactness assumption (T1), the following lemma.

Lemma 3 Let $\lambda=(a, b) \in \Lambda$. Then

$$
\operatorname{Var}\left(P_{T_{\lambda}} f\right) \leq \frac{2}{a} \operatorname{Var}(f)+2\left|\int f d m\right| \quad \text { for all } f \in B V \text { and } n \in \mathbb{N} .
$$

(This implies immediately Hypothesis 1 with $C_{1}=1+\frac{a_{0}}{a_{0}-2}$ and Hypothesis $\mathbf{Q}$ as well as its strengthening (29) with $\gamma=\frac{2}{a_{0}}$ and $C_{2}=C_{2}^{\prime}=1$.)

\footnotetext{
${ }^{2}$ Indeed, Hofbauer shows this for the maximal measure of such maps, but since these maps have constant slope, the maximal measures are just the absolutely continuous ones. For numerical results on the probability densities associated with these measures and how they change under parameter variation see [27].
} 
Proof: Denote by $\mathcal{F}$ the family of all $C^{1}$-functions $\varphi: \mathbb{R} \rightarrow \mathbb{R}$ with $|\varphi|_{\infty} \leq 1$. In [23, eq. (11)] a number $\Gamma(\varphi, s) \geq 0$ is defined for each pair of $\varphi \in \mathcal{F}$ and $s \in \mathbb{R}$. In view of [23, eq. (13) and (14)] it suffices to show that for each $\varphi \in \mathcal{F}$ there is some $s \in[-2,2]$ such that $\Gamma(\varphi, s) \leq \frac{2}{a} \sqrt[3]{4}$ We will show that this is the case for $s=s_{\varphi}:=\varphi\left(\frac{1}{2}\right)-\varphi\left(-\frac{1}{2}\right)$.

Let $p=\left\lceil\frac{a+1}{2}-b\right\rceil-1$ and $q=\left\lceil\frac{a+1}{2}+b\right\rceil-1$. Then $-p-\frac{1}{2} \leq T_{\lambda}\left(-\frac{1}{2}\right)<-p+\frac{1}{2}$, $q-\frac{1}{2}<T_{\lambda}\left(\frac{1}{2}\right) \leq q+\frac{1}{2}$, and $T_{\lambda}$ has monotonicity intervals $\left(A_{k}, B_{k}\right), k=-p, \ldots, q$, where

$$
\begin{array}{lll}
A_{-p}=-\frac{1}{2}, & A_{k}=a^{-1}\left(k-\frac{1}{2}-b\right) & (k=-p+1, \ldots, q) \\
B_{q}=\frac{1}{2}, & B_{k}=a^{-1}\left(k+\frac{1}{2}-b\right) & (k=-p, \ldots, q-1)
\end{array}
$$

In order to estimate $\Gamma(\varphi, s)$ in [23, eq. (11)] one has to evaluate certain terms $U_{k}:=\psi\left(A_{k}\right)-$ $\ell_{k} g_{k}\left(A_{k}\right)-s A_{k}$ and $V_{k}:=\psi\left(A_{k}\right)-\ell_{k} g_{k}\left(A_{k}\right)-s B_{k}$. In our case, $g_{k}\left(A_{k}\right)=a^{-1}$, as $g_{k}$ is the inverse of the derivative of the $k$-th monotone branch. The quantity $\ell_{k}$ is an abbreviation for $\varphi\left(T_{\lambda} A_{k}\right)$, where $T_{\lambda} A_{k}$ denotes a limit from the right. Therefore, using the formula on the bottom of [23, p.1779], we obtain

$$
\begin{aligned}
a U_{k} & =a \cdot\left(\psi\left(A_{k}\right)-\ell_{k} g_{k}\left(A_{k}\right)-s_{\varphi} A_{k}\right) \\
& =-\varphi\left(-\frac{a}{2}+b+p\right)+(k+p)\left(\varphi\left(\frac{1}{2}\right)-\varphi\left(-\frac{1}{2}\right)\right)-s_{\varphi} \cdot\left(k-\frac{1}{2}-b\right) \\
& =-\varphi\left(-\frac{a}{2}+b+p\right)+s_{\varphi} \cdot\left(p+\frac{1}{2}+b\right) \quad \text { if } k>-p, \\
a U_{-p} & =-\varphi\left(-\frac{a}{2}+b+p\right)+s_{\varphi} \cdot \frac{a}{2},
\end{aligned}
$$

and similarly,

$$
\begin{aligned}
a V_{k}= & a \cdot\left(\psi\left(A_{k}\right)-\ell_{k} g_{k}\left(A_{k}\right)-s_{\varphi} B_{k}\right) \\
& =-\varphi\left(-\frac{a}{2}+b+p\right)+s_{\varphi} \cdot\left(p-\frac{1}{2}+b\right) \quad \text { if } k<q, \\
a V_{q}= & -\varphi\left(-\frac{a}{2}+b+p\right)+s_{\varphi} \cdot\left(p+q-\frac{a}{2}\right) .
\end{aligned}
$$

It follows that $\left|V_{k}-U_{k^{\prime}}\right| \leq a^{-1}\left|s_{\varphi}\right|$ for all $k, k^{\prime} \in\{-p, \ldots, q\}$. Hence, by [23, eqs. (11) and (12)],

$$
\Gamma\left(\varphi, s_{\varphi}\right) \leq a^{-1}+\frac{1}{2} a^{-1}\left|s_{\varphi}\right| \leq \frac{2}{a} .
$$

q.e.d.

Next we check assumption (T2) on the Lipschitz dependence of the maps on the parameters, so we estimate $d\left(T_{a, b}, T_{a^{\prime}, b^{\prime}}\right)$. For the proof we extend the maps to the whole real line (keeping the same names) by applying definition (34) to all $x \in \mathbb{R}$.

Suppose $a^{\prime}<a$ and denote by $A_{k}$ and $A_{k}^{\prime}, k=-p+1, \ldots, q$, the discontinuity points of the two maps as introduced in the proof of Lemma 3. Consider the linear map $L: \mathbb{R} \rightarrow \mathbb{R}$, $L(x)=\left(a x+b-b^{\prime}\right) / a^{\prime}$ and observe that $L\left(A_{k}\right)=A_{k}^{\prime}(-p<k \leq q)$ and $a^{\prime} L(x)+b^{\prime}=a x+b$ for all $x \in \mathbb{R}$. Let $[u, v]:=I \cap L^{-1}(I)$ and $I_{0}:=[u+\delta, v-\delta]$ for some arbitrarily small $\delta>0$. Define $\sigma: I \rightarrow I$ by $\sigma(x)=L(x)$ if $x \in I_{0}$ and extend $\sigma$ to a diffeomorphism of $I$. Then

- $m\left(I \backslash I_{0}\right) \leq\left(1-\frac{a^{\prime}}{a}\right)+\left|b^{\prime}-b\right| / a+2 \delta \leq a_{0}^{-1}\left(\left|a^{\prime}-a\right|+\left|b^{\prime}-b\right|\right)+2 \delta$

- $|\sigma(x)-x|=|L(x)-x| \leq \frac{1}{2}\left|\frac{a}{a^{\prime}}-1\right|+\left|b-b^{\prime}\right| / a^{\prime} \leq a_{0}^{-1}\left(\frac{1}{2}\left|a-a^{\prime}\right|+\left|b-b^{\prime}\right|\right)$ for all $x \in I_{0}$

- $\left|1 / \sigma^{\prime}(x)-1\right|=\left|\frac{a^{\prime}}{a}-1\right| \leq a_{0}^{-1}\left|a^{\prime}-a\right|$ for all $x \in I_{0}$

- $T_{a^{\prime}}(\sigma(x))=T_{a}(x)$ for all $x \in I_{0}$.

So $d\left(T_{a}, T_{a^{\prime}}\right) \leq a_{0}^{-1}\left(\left|a-a^{\prime}\right|+\left|b-b^{\prime}\right|\right)$ as $\delta>0$ could be chosen arbitrarily small,

\footnotetext{
${ }^{3} \operatorname{var}($.$) in [23$ is the same as $\operatorname{Var}($.$) here. This is different from the use of \operatorname{var}($.$) in [25].$

${ }^{4}$ Following this reference precisely, the reader will notice that instead of the factor $\frac{2}{a}$ one gets the factor $\frac{2}{a}+\frac{1}{2} V_{g}$. But $V_{g}=0$ for piecewise linear transformations as noticed at the bottom of [23 p.1779].
} 


\section{Transport coefficients}

We apply the results of the previous sections to determine transport coefficients of the deterministic random walks generated by the maps $T_{\lambda}=T_{a, b}$ from subsection 3.2. The random walks in question are $S_{\lambda, n}=\sum_{k=0}^{n-1} \psi_{\lambda} \circ T_{\lambda}^{k}$ with

$$
\psi_{\lambda}(x)=(a-1) x+b .
$$

It is an easy exercise to see that Hypothesis 3 and 4 as well as their strengthening (30) are satisfied: $\operatorname{Var}\left(\psi_{a, b}\right) \leq 2(a-1+|b|)<2\left(a_{1}+1\right)=: C_{3}$ and $\left|\psi_{a, b}-\psi_{a^{\prime}, b^{\prime}}\right|_{1} \leq \frac{1}{2} \operatorname{Var}\left(\psi_{a, b}-\psi_{a^{\prime}, b^{\prime}}\right) \leq$ $\left|a-a^{\prime}\right|+\left|b-b^{\prime}\right|$.

For later use we note that the maps $T_{a, b}$ and $T_{a,-b}$ are conjugate in the sense that $T_{a, b}(-x)=$ $-T_{a,-b}(x)$, in particular $h_{a,-b}(-x)$ is also an invariant density for $T_{a, b}$ and, by uniqueness, $h_{a, b}(x)=h_{a,-b}(-x)$.

We first note the following explicit form of the drift:

$$
J(\lambda)=J(a, b):=\int \psi_{a, b} h_{a, b} d m=b+(a-1) \int x h_{a, b}(x) d x .
$$

As noted above, $h_{a, 0}(x)=h_{a, 0}(-x)$. Hence $J(a, 0)=05$

\subsection{Upper bounds for the modulus of continuity of the drift and the diffusion coefficient}

Now we apply Corollary 1 and Proposition 1 to our setting.

Proposition 2 For the family of maps $\left(T_{\lambda}: \lambda \in \Lambda\right)$ defined above, there are constants $K_{3}, K_{4}>$ 0 such that the drift $J(\lambda):=J\left(T_{\lambda}\right)$ and the diffusion coefficient $D(\lambda):=D\left(T_{\lambda}\right), \lambda=(a, b)$, satisfy

$$
\begin{gathered}
\left|J(\lambda)-J\left(\lambda^{\prime}\right)\right| \leq K_{3} \cdot\left|\lambda-\lambda^{\prime}\right| \cdot\left(1+|\log | \lambda-\lambda^{\prime}||\right) \quad\left(\lambda, \lambda^{\prime} \in \Lambda\right) \\
\left|D(\lambda)-D\left(\lambda^{\prime}\right)\right| \leq K_{4} \cdot\left|\lambda-\lambda^{\prime}\right| \cdot\left(1+|\log | \lambda-\lambda^{\prime}||\right)^{2} \quad\left(\lambda, \lambda^{\prime} \in \Lambda\right) .
\end{gathered}
$$

Corollary 2 a) The graph of $D: \Lambda \rightarrow \mathbb{R}$ has box- and Hausdorff-dimension 2 .

b) For each $b \in \mathbb{R}$, the graph of $D_{b}:\left[a_{0}, a_{1}\right] \rightarrow \mathbb{R}, D_{b}(a)=D(a, b)$, has box- and Hausdorffdimension 1 .

c) For each $a>2$, the graph of $D_{a}:\left[-\frac{1}{2}, \frac{1}{2}\right] \rightarrow \mathbb{R}, D_{a}(b)=D(a, b)$, has box- and Hausdorffdimension 1 .

Proof: Denote by $\operatorname{dim}_{B}$ and $\operatorname{dim}_{H}$ the box and Hausdorff dimension, respectively. Obviously, $2 \leq \operatorname{dim}_{H}(\operatorname{graph}(D)) \leq \operatorname{dim}_{B}(\operatorname{graph}(D))$. So it remains to show that $\operatorname{dim}_{B}(\operatorname{graph}(D)) \leq 2$. To this end subdivide the rectangle $\Lambda$ into little squares of equal size $\approx N^{-1}$. For each such square $Q$ we have

$$
\max \{D(\lambda): \lambda \in Q\}-\min \{D(\lambda): \lambda \in Q\} \leq K_{4} N^{-1}(1+\log N)^{2} .
$$

Hence,

$$
\operatorname{dim}_{B}(\operatorname{graph}(D)) \leq \limsup _{N \rightarrow \infty} \frac{\log \left(K_{4} N^{2}(1+\log N)^{2}\right)}{\log N}=2 .
$$

The two other claims are proved in the same way.

q.e.d.

\footnotetext{
${ }^{5}$ In [27] it was conjectured, based on analyzing these deterministic random walks in terms of Markov partitions, that for $b=0$ and $a>2$ the maps $T_{\lambda}$ exhibit a central limit theorem and that a diffusion coefficient exists, which is confirmed by (17).
} 
Remark 3 Corollary 2 has already been conjectured by Koza [35. His conjecture was based on calculating the pointwise Minkowski-Bouligand dimension for algebraic Markov partition parameter values of this family of maps by using the exact solutions for drift and diffusion coefficient given in [15. This led him to conclude that the oscillation 41] of $D(\lambda)$ is linear in the size of the subinterval multiplied with a logarithmic term, cp. (20) of [35] with (43) above. The exponent of this logarithmic correction was found to be either one or two depending on the type of Markov partition.

\subsection{A closer look at maps with integer slope}

We finish this section with a closer look at the functions $D_{a}(b)$ when $a$ is an integer larger than 2. In this case $T=T_{a, b}$ can be seen as an $a$-fold covering linear circle map, so it leaves Lebesgue measure invariant. Therefore $h_{T}=1$ for all such $T$ and the estimates from the proof of Proposition 1 simplify drastically: Fix $a \in\{3,4,5, \ldots\}$. Then $J(a, b)=b$ for all $b$ (see (40) and [15, 32] $), \hat{\psi}_{a, b}(x)=(a-1) x=: \hat{\psi}_{a}(x)$ is the same for all $b$, and denoting $T_{1}=T_{a, b}$ and $T_{2}=T_{a, b^{\prime}}$ we can replace estimates (22) - (27) by

$$
\begin{aligned}
& \int_{I} P_{T_{2}}^{n}\left(\hat{\psi}_{T_{2}} h_{T_{2}}\right) \hat{\psi}_{T_{2}} d m-\int_{I} P_{T_{1}}^{n}\left(\hat{\psi}_{T_{1}} h_{T_{1}}\right) \hat{\psi}_{T_{1}} d m \\
= & \int_{I} \hat{\psi}_{a}(x) \hat{\psi}_{a}\left(T_{2}^{n} x\right) d x-\int_{I} \hat{\psi}_{a}(x) \hat{\psi}_{a}\left(T_{1}^{n} x\right) d x \\
= & (a-1)^{2}\left(\int_{I} x T_{a, b^{\prime}}^{n}(x) d x-\int_{I} x T_{a, b}^{n}(x) d x\right)
\end{aligned}
$$

To evaluate this difference assume henceforth that $0 \leq b \leq \frac{1}{2}$. There is no loss in doing so, because $T_{a, b}$ and $T_{a,-b}$ are conjugate as obseved above. Define the "rotation" $R: I \rightarrow I$ by $R(x)=x-\frac{b}{a-1} \bmod \left(\mathbb{Z}-\frac{1}{2}\right)$. It conjugates $T_{a, b}$ to $T_{a, 0}$, namely

$$
R\left(T_{a, 0}^{n} x\right)=T_{a, b}^{n}(R x) \quad \text { for all } x \in I \text { and } n \in \mathbb{N} .
$$

Therefore, denoting $\hat{b}=-\frac{1}{2}+\frac{b}{a-1}$ and $\chi_{b}(x)=1_{\left[-\frac{1}{2}, \hat{b}\right)}(x)-\frac{b}{a-1}$,

$$
\int_{I} x T_{a, b}^{n}(x) d x=\int_{I} R(x) R\left(T_{a, 0}^{n} x\right) d x=\int_{I}\left(x+\chi_{b}(x)\right)\left(T_{a, 0}^{n} x+\chi_{b}\left(T_{a, 0}^{n} x\right)\right) d x
$$

so that

$$
\begin{aligned}
& \int_{I} x T_{a, b^{\prime}}^{n}(x) d x-\int_{I} x T_{a, b}^{n}(x) d x \\
= & \int_{I} P_{a, 0}^{n} x \cdot\left(\chi_{b^{\prime}}(x)-\chi_{b}(x)\right) d x+\int_{I} P_{a, 0}^{n}\left(\chi_{b^{\prime}}-\chi_{b}\right)(x) \cdot x d x \\
& +\int_{I} P_{a, 0}^{n} \chi_{b^{\prime}}(x) \cdot\left(\chi_{b^{\prime}}(x)-\chi_{b}(x)\right) d x+\int_{I} P_{a, 0}^{n}\left(\chi_{b^{\prime}}-\chi_{b}\right)(x) \cdot \chi_{b}(x) d x
\end{aligned}
$$

As $\int_{I} \chi_{b^{\prime}}(x) d x=0$ and $\operatorname{Var}\left(\chi_{b}\right)=2$ for all $b$, the third term is of order $\mathcal{O}\left((2 / a)^{n}\left|b^{\prime}-b\right|\right)$ by Lemma 3. As $P_{a, 0}^{n} x=a^{-n} x$, the first term is at most of the same order. Therefore their sums over all $n$ are of the order $\mathcal{O}\left(\left|b^{\prime}-b\right|\right)$.

We turn to the two remaining terms. Their sum from $n=0$ to $\infty$ is of the form

$$
\sum_{n=0}^{\infty} \int_{I} P_{a, 0}^{n}\left(\chi_{b^{\prime}}-\chi_{b}\right)(x) \cdot g_{b}(x) d x=\sum_{n=0}^{\infty} \int_{I}\left(\chi_{b^{\prime}}-\chi_{b}\right)(x) \cdot g_{b}\left(T_{a, 0}^{n} x\right) d x
$$

with $g_{b}(x)=x+\chi_{b}(x)$. Let $\delta=\frac{b^{\prime}-b}{a-1}$. As $\int_{-1 / 2}^{1 / 2}\left(\chi_{b^{\prime}}-\chi_{b}\right)(x) d x=0, \operatorname{Var}\left(\chi_{b^{\prime}}-\chi_{b}\right) \leq 4$, and $\left|g_{b}\right| \leq 2$, the $n$-th integral is of order $\mathcal{O}\left((2 / a)^{n}\right)$. Hence the sum from $n=N_{\delta}:=\left\lceil\frac{\log |\delta|^{-1}}{\log a}\right\rceil$ to 
$\infty$ is of order $|\delta|$, and it remains to estimate the sum from $n=0$ to $N_{\delta}-1$. For these $n$ we have $a^{n}|\delta| \leq 1$.

We start with the special case $b=0$ where we have $\chi_{b}=0$, so $g_{b}(x)=x$. Then the $n$-th term in the sum (46) evaluates to

$$
\int_{-\frac{1}{2}}^{-\frac{1}{2}+\delta} T_{a, 0}^{n}(x) d x= \begin{cases}\delta \cdot\left(-\frac{1}{2}+\frac{1}{2} a^{n} \delta\right) & \text { if } a \text { is odd } \\ \delta \cdot\left(\frac{1}{2} a^{n} \delta\right) & \text { if } a \text { is even and } n \geq 1\end{cases}
$$

It follows that the sum from $n=0$ to $N_{\delta}-1$ in (46) is of the order $\mathcal{O}(|\delta|)$ if $a$ is even and that it is $\delta \cdot\left(-\frac{\log |\delta|^{-1}}{2 \log a}+\mathcal{O}(1)\right)$ if $a$ is odd.

Consider next the case $b=\frac{1}{2}$. As $b=-\frac{1}{2}$ gives rise to the same map, the we may assume w.l.o.g. that $-\frac{1}{2}<b^{\prime}<b$, i.e. $\delta<0$. If $a$ is odd, then $T_{a, 0} \hat{b}=\hat{b}+\frac{1}{2}$ and $T_{a, 0}\left(\hat{b}+\frac{1}{2}\right)=\hat{b}$. Therefore

$$
\begin{aligned}
\int_{I}\left(\chi_{b^{\prime}}-\chi_{b}\right)(x) \cdot g_{b}\left(T_{a, 0}^{n} x\right) d x & =\int_{\hat{b}}^{\hat{b}^{\prime}} g_{b}\left(T_{a, 0}^{n} x\right) d x \\
& = \begin{cases}\int_{0}^{\delta}\left(\hat{b}+a^{n} t\right)+\chi_{b}\left(\hat{b}+a^{n} t\right) d t & \text { if } n \text { is even } \\
\int_{0}^{\delta}\left(\hat{b}+\frac{1}{2}+a^{n} t\right)+\chi_{b}\left(\hat{b}+\frac{1}{2}+a^{n} t\right) d t & \text { if } n \text { is odd }\end{cases} \\
& = \begin{cases}\delta \hat{b}+\frac{1}{2} a^{n} \delta^{2}+\delta-\delta \frac{b}{a-1} & \text { if } n \text { is even } \\
\delta\left(\hat{b}+\frac{1}{2}\right)+\frac{1}{2} a^{n} \delta^{2}-\delta \frac{b}{a-1} & \text { if } n \text { is odd }\end{cases}
\end{aligned}
$$

as long as $a^{n}|\delta|<\frac{b}{a-1}$, i.e. $n<\tilde{N}_{\delta}:=N_{\delta}-\frac{\log (a-1)-\log b}{\log a}$. For the remaining $n$ this identity needs to be modified by at most $|\delta|$. In any case,

$\sum_{n=0}^{N_{\delta}-1} \int_{I}\left(\chi_{b^{\prime}}-\chi_{b}\right)(x) \cdot g_{b}\left(T_{a, 0}^{n} x\right) d x=\frac{N_{\delta}}{2} \cdot \frac{\delta}{2}+\frac{N_{\delta}}{2} \cdot 0+\mathcal{O}(\delta)=\delta \log |\delta|^{-1} \frac{1}{4 \log a}+\mathcal{O}(\delta) \quad$ for odd $a$

with a constant in "O $O$ " that depends on $b$ and $a$ but not on $b^{\prime}$. If $a$ is even, then $T_{a, 0} \hat{b}=\hat{b}$, and following the argument for odd $a$ and even $n$ we obtain

$$
\sum_{n=0}^{N_{\delta}-1} \int_{I}\left(\chi_{b^{\prime}}-\chi_{b}\right)(x) \cdot g_{b}\left(T_{a, 0}^{n} x\right) d x=N_{\delta} \cdot \frac{\delta}{2}+\mathcal{O}(\delta)=\delta \log |\delta|^{-1} \frac{1}{2 \log a}+\mathcal{O}(\delta) \quad \text { for even } a .
$$

We turn to more general parameters $0<b<\frac{1}{2}$. We have to estimate

$$
\begin{aligned}
s(\delta) & :=\sum_{n=0}^{N_{\delta}-1} \int_{I}\left(\chi_{b^{\prime}}-\chi_{b}\right)(x) \cdot g_{b}\left(T_{a, 0}^{n} x\right) d x=\sum_{n=0}^{N_{\delta}-1} \int_{\hat{b}}^{\hat{b}+\delta} g_{b}\left(T_{a, 0}^{n} x\right) d x \\
& =\sum_{n=0}^{N_{\delta}-1} \int_{0}^{\delta} g_{b}\left(T_{a, 0}^{n}(\hat{b})+a^{n} t\right) d t
\end{aligned}
$$

The details of this estimate depend strongly on the distributional properties of the orbit of $\hat{b}$ under $T_{a, 0}$ and we discuss only two particular but important cases where the situation does not become too complicated. First we look at such $b$ for which the orbit of $\hat{b}$ is eventually periodic but where the periodic part does neither contain $-\frac{1}{2}$ nor $\hat{b}$. (This is a countable dense set of parameters.) In this case one can argue as above for $b=\frac{1}{2}$ and odd $a$ and show that

$$
s(\delta)=\delta \log |\delta|^{-1} \frac{C_{b}}{\log a}+\mathcal{O}(\delta)
$$

with $C_{b}=\int_{I} g_{b}(x) d \mu_{b}(x)$ where $\mu_{b}$ is the equidistribution on the periodic part of the orbit of $\hat{b}$. Exceptionally this may be zero, but typically it won't. 
Next we look at Lebesgue typical points $\hat{b}$, i.e. at Lebesgue typical parameters $b$. For fixed $\delta$ we interpret $s(\delta)$ as a random variable where randomness is introduced via the parameter $b \in\left(0, \frac{1}{2}\right)$. We are going to show that the random variables $\delta^{-1} N_{\delta}^{-\frac{1}{2}} s(\delta)$ converge in distribution to a mixture of Gaussians, i.e.

$$
\mathcal{L}\left(\delta^{-1} N_{\delta}^{-\frac{1}{2}} s(\delta)\right) \Rightarrow \int_{0}^{1} \mathcal{N}\left(0, \sigma_{z}^{2}\right) d z \quad \text { as } \delta \rightarrow 0
$$

with suitable variances $\sigma_{z}^{2}>0$ that depend on the fixed parameter $a$. This shows that approximately $s(\delta)=\delta\left(\log |\delta|^{-1}\right)^{\frac{1}{2}} Z$ with a random variable $Z$ that is a mixture of Gaussians which depends only on the fixed integer parameter $a$.

As a first step we compare $s(\delta)$ to $\sum_{n=0}^{N_{\delta}-1} \delta \cdot g_{b}\left(T_{a, 0}^{n} \hat{b}\right)$. As $g_{b}(x)=x+\chi_{b}(x)$, we can estimate the difference for the $x$ - and the $\chi_{b}(x)$-constributions separately. For the $x$-contribution the difference is easily seen to be of order $\mathcal{O}(\delta)$. For the $\chi_{b}(x)$-contribution we estimate each of the last $L_{\delta}:=\left\lceil\frac{3}{\log a} \log N_{\delta}\right\rceil$ terms of the sum by 2 thus getting a contribution of order $\mathcal{O}\left(\delta \log N_{\delta}\right)$. For the remaining terms we note the following two estimates which are obvious from a short look at the graph of $T_{a, 0}^{n}$ :

$$
m\left\{b \in I:\left|T_{a, 0}^{n}(\hat{b})-\left(-\frac{1}{2}\right)\right|<a^{n} \delta\right\}, m\left\{b \in I:\left|T_{a, 0}^{n}(\hat{b})-\hat{b}\right|<a^{n} \delta\right\} \leq 4 a^{n} \delta,
$$

so that

$$
\begin{aligned}
& m\left\{b \in I: \exists n \in\left\{0, \ldots, N_{\delta}-L_{\delta}-1\right\} \text { s.th. }\left|T_{a, 0}^{n}(\hat{b})-\left(-\frac{1}{2}\right)\right|<a^{n} \delta \text { or }\left|T_{a, 0}^{n}(\hat{b})-\hat{b}\right|<a^{n} \delta\right\} \\
& \leq \frac{8}{a-1} a^{-L_{\delta}}=\frac{8}{a-1} N_{\delta}^{-3} .
\end{aligned}
$$

It follows that $\sum_{n=0}^{N_{\delta}-L_{\delta}-1} \int_{0}^{\delta} \chi_{b}\left(T_{a, 0}^{n}(\hat{b})+a^{n} t\right) d t=\sum_{n=0}^{N_{\delta}-L_{\delta}-1} \delta \chi_{b}\left(T_{a, 0}^{n} \hat{b}\right)$ except on a set of $b$ of Lebesgue measure at most $\frac{8}{a-1} N_{\delta}^{-3}$. Hence, observing that $L_{\delta} N_{\delta}^{-1} \rightarrow 0$ as $\delta \rightarrow 0$, the convergence in (48) will follow once we have proved

$$
\mathcal{L}\left(Y_{N_{\delta}}\right) \Rightarrow \int_{0}^{1} \mathcal{N}\left(0, \sigma_{z}^{2}\right) d z \quad \text { as } N_{\delta} \rightarrow \infty
$$

where $Y_{N}(b):=N^{-\frac{1}{2}} \sum_{n=0}^{N-1} g_{b}\left(T^{n} \hat{b}\right)$ and $b$ is uniformly distributed in the interval $\left(0, \frac{1}{2}\right)$. (To ease the notation we abbreviate $T_{a, 0}$ by $T$.) As the single contributions to the sum in $Y_{N}$ depend on $b$ via $T^{n}(\hat{b})$ and $b$ itself, this is not the situation of the usual central limit theorem, so we treat the problem in two steps:

Step 1: For fixed $z \in(0,1)$ consider $Y_{N}^{z}(b):=N^{-\frac{1}{2}} \sum_{n=0}^{N-1} g_{z}\left(T^{n} \hat{b}\right)$. It is a well known general fact that, for fixed $z$, the $Y_{N}^{z}$ converge in distribution to some $\mathcal{N}\left(0, \sigma_{z}^{2}\right)$ (see e.g. [21, 18, 38]) - except for the strict positivity of $\sigma_{z}^{2}$. To prove this we use [38, Lemma 6]: suppose for a contradiction that $\sigma_{z}^{2}=0$. Then there is a function $\psi: I \rightarrow \mathbb{R}$ of bounded variation such that $g_{z}(x)=\psi(T x)-\psi(x)$ for Lebesgue-a.e. $x$. Let $M:=2 \sup |\psi|$. Then $\left|\sum_{k=0}^{n} g_{z}\left(T^{k} x\right)\right| \leq M$ for all $n$ and a.a. $x$. Looking at suitable periodic orbits it is easy to see that there are $n=n_{0}$ and $x=x_{0}$ for which this sum is larger than $M+2$. But then, as both $T$ and also $g_{z}$ are at least one-sided continuous, there is a small interval close to $x_{0}$ on which the same sum is larger than $M+1$, which contradicts the above bound that holds for all $n$ and Lebesgue-a.a. $x$.

Step 2: We need a number of preparations:

(i) Let $J:=\left(-\frac{1}{2},-\frac{1}{2}+\frac{1}{2(a-1)}\right)$ be the interval through which $\hat{b}$ ranges when $b$ is chosen randomly from $\left(0, \frac{1}{2}\right)$.

(ii) Let $\left(r_{j}\right)_{j}>0$ be any sequence of natural numbers tending to infinity and such that $r_{j} \leq j^{\frac{1}{4}}$ for all $j$. For each $j$ denote by $C_{j} \subset I=\left[-\frac{1}{2}, \frac{1}{2}\right]$ a set of points that subdivides $I$ into $a^{r_{j}}$ intervals of the same length which are mapped onto $I$ bijectively by $T^{r_{j}}$. (If $a$ is odd take $C_{j}:=T^{-r_{j}}\left\{-\frac{1}{2}\right\}$, if $a$ is even take $C_{j}:=T^{-r_{j}}\{0\}$.) For $z \in C_{j}$ denote by $I_{z}^{j}$ the subinterval with left endpoint $z$. 
(iii) $\left\|Y_{N}-Y_{N} \circ T^{2 r_{N}}\right\|_{\infty}=\mathcal{O}\left(r_{N} N^{-\frac{1}{2}}\right)=o(1)$ as $N \rightarrow \infty$ because the two sums involved differ only by $4 r_{N}$ terms.

(iv) $\operatorname{Var}\left(P_{T}^{r_{N}}\left(\frac{1}{m(J)} 1_{J}-1\right)\right) \leq\left(\frac{2}{a}\right)^{r_{N}} \operatorname{Var}\left(\frac{1}{m(J)} 1_{J}-1\right)=o(1)$ as $N \rightarrow \infty$ by Lemma 3 ,

(v) $m\left\{x \in I_{z}^{N}: Y_{N}^{z}\left(T^{r_{N}} x\right) \neq Y_{N}\left(T^{r_{N}} x\right)\right\}=a^{-r_{N}} m\left\{x \in I: Y_{N}^{z}(x) \neq Y_{N}(x)\right\} \leq a^{-r_{N}} m\left(I_{z}^{N}\right)$.

In order to prove (48) we now proceed as follows: it suffices to show that for each bounded Lipschitz function $\phi: \mathbb{R} \rightarrow \mathbb{R}$ holds

$$
\frac{1}{m(J)} \int_{J} \phi\left(Y_{N}(b)\right) d b=\int_{0}^{1}\left(\int_{I} \phi(b) d \mathcal{N}\left(0, \sigma_{z}^{2}\right)(b)\right) d z+o(1) \quad \text { as } N \rightarrow \infty .
$$

To simplify the notation we write $\int_{J} \phi\left(Y_{N}\right) d m$ instead of $\int_{J} \phi\left(Y_{N}(b)\right) d b$ etc..

$$
\begin{aligned}
\frac{1}{m(J)} \int_{J} \phi\left(Y_{N}\right) d m & =\frac{1}{m(J)} \int_{J} \phi\left(Y_{N} \circ T^{2 r_{N}}\right) d m+o(1) \quad \text { by (iii) } \\
& =\int_{I} P_{T}^{r_{N}}\left(m(J)^{-1} 1_{J}\right) \cdot \phi\left(Y_{N} \circ T^{r_{N}}\right) d m+o(1) \\
& =\int_{I} \phi\left(Y_{N} \circ T^{r_{N}}\right) d m+o(1) \quad \text { by (iv) } \\
& =\sum_{z \in C_{N}} \int_{I_{z}^{N}} \phi\left(Y_{N}^{z} \circ T^{r_{N}}\right) d m+o(1) \quad \text { by (v) } \\
& =\sum_{z \in C_{N}} \int_{I} P_{T}^{r_{N}} 1_{I_{z}^{N}} \cdot \phi\left(Y_{N}^{z}\right) d m+o(1) \\
& =\sum_{z \in C_{N}} m\left(I_{z}^{N}\right) \int_{I} \phi\left(Y_{N}^{z}\right) d m+o(1) \\
& =\sum_{z \in C_{N}} m\left(I_{z}^{N}\right) \int_{I} \phi d \mathcal{N}\left(0, \sigma_{z}^{2}\right)+o(1) \quad \text { by step } 1 \\
& =\int_{0}^{1}\left(\int_{I} \phi d \mathcal{N}\left(0, \sigma_{z}^{2}\right)\right) d z+o(1) \quad \text { as } N \rightarrow \infty
\end{aligned}
$$

where one has to choose a sufficiently slowly growing sequence $\left(r_{N}\right)$ in the second last equality.

Summary of results for integer $a$ In view of the factor $(a-1)^{2}$ in (45) and of the definition of $\delta=\frac{b^{\prime}-b}{a-1}$ the above discussion shows:

(1) For even $a \geq 4$,

$$
\begin{aligned}
D_{a}\left(b^{\prime}\right)-D_{a}(0) & =\mathcal{O}\left(b^{\prime}\right) \quad \text { and } \\
D_{a}\left(b^{\prime}\right)-D_{a}\left(\frac{1}{2}\right) & =\frac{a-1}{2 \log a}\left(b^{\prime}-\frac{1}{2}\right) \log \left|b^{\prime}-\frac{1}{2}\right|^{-1}+\mathcal{O}\left(b^{\prime}-\frac{1}{2}\right)
\end{aligned}
$$

(2) For odd $a \geq 3$,

$$
\begin{aligned}
& D_{a}\left(b^{\prime}\right)-D_{a}(0)=-\frac{a-1}{2 \log a} b^{\prime} \log \left|b^{\prime}\right|^{-1}+\mathcal{O}\left(b^{\prime}\right) \quad \text { and } \\
& D_{a}\left(b^{\prime}\right)-D_{a}\left(\frac{1}{2}\right)=\frac{a-1}{4 \log a}\left(b^{\prime}-\frac{1}{2}\right) \log \left|b^{\prime}-\frac{1}{2}\right|^{-1}+\mathcal{O}\left(b^{\prime}-\frac{1}{2}\right)
\end{aligned}
$$


(3) If $b$ is such that $\hat{b}=-\frac{1}{2}+\frac{b}{a-1}$ is eventually periodic under $T_{a, 0}$ and the periodic part of the orbit neither contains $-\frac{1}{2}$ nor $\hat{b}$, then there is a constant $C_{a, b}$ such that

$$
D_{a}\left(b^{\prime}\right)-D_{a}(b)=C_{a, b}\left(b^{\prime}-b\right) \log \left|b^{\prime}-b\right|^{-1}+\mathcal{O}\left(b^{\prime}-b\right) .
$$

This generalizes observations (11) and (2). See also Remark 3 .

(4) For fixed $\delta$ and random $b$ drawn uniformly from $\left(0, \frac{1}{2}\right)$ or from $\left(-\frac{1}{2}, 0\right)$,

$$
D_{a}(b+\delta(a-1))-D_{a}(b)=C_{a} \delta\left(\log |\delta|^{-1}\right)^{1 / 2} Z_{\delta}+\mathcal{O}(\delta) \text { as } \delta \rightarrow 0
$$

with a constant $C_{a}>0$ and random variables $Z_{\delta}$ which all have the same distribution - a mixture of Gaussians as in (48) depending only on the fixed parameter $a$.

In view of these findings the graphs of $D_{a}:\left[-\frac{1}{2}, \frac{1}{2}\right] \rightarrow \mathbb{R}$ are fractal in the sense of Section 12.2 in [41 - at least for integer values of $a$-, although they have box - and Hausdorff-dimension 1.

Remark 4 In Refs. 27, 14, 32 it has been shown that for $b=0$ the dynamics of $\psi_{\lambda}$ can be expressed in terms of generalized Takagi (or de Rham) functions. Analogous conclusions hold for the case of $b \neq 0$ [34. The above results are thus intimately related to continuity properties of this class of functions under parameter variation. These functions are defined by simple functional recursion relations and have been introduced in the literature completely independently from the diffusion problem considered here.

\section{$5 \quad$ Numerical results}

Guided by the analytical results of the previous sections, in this part we numerically study the transport coefficients generated by the piecewise linear maps (34). We are aiming particularly at a numerical verification of Proposition 2 and of the summary of Section 4.2. To some extent the numerics enables us to go beyond the analytical findings as far as detailed local properties of these transport coefficients are concerned.

Let us start with a reminder of previous results: Exact analytical solutions for drift $J$ and diffusion coefficient $D$ for all parameter values $a, b$ were derived in [15] 6 In [30] data sets were generated from these expressions and analyzed by standard numerical box counting [41]. This procedure relies on the assumption that

$$
N(\epsilon) \sim \epsilon^{-B}
$$

for small enough $\epsilon$, where $N$ is the number of square boxes of side length $\epsilon$ needed to cover the graph of $J$ or $D$, and $B=\operatorname{dim}_{B}$ (graph) defines the box (counting) dimension. Analysing $D(a)=D(a, 0)$ on $2 \leq a \leq 8$, see Fig. 1 (a), based on $10^{6}$ data points uniformly distributed in $a$ yielded a box dimension of $B \simeq 1.039$ [30]. The inset in Fig. 1] (a) depicts $N(\epsilon)$ for the new, larger data set of $10^{9}$ points of $D(a)$ in comparison to (51) with the above exponent. Data and fit are undistinguishable.

Fig. 1 (b) displays the numerical results from [30] for the local box dimension $B(a)$ of $D(a)$. That is, according to (51) $B$ was computed locally on a regular grid of small subintervals $\Delta a$ centered around $a$. The figure shows that (51) yields locally different results for $B(a)$ forming an oscillatory structure that becomes more pronounced the smaller $\Delta a$. Consequently, $D(a)$ was said to be characterized by a "fractal fractal dimension" in [30].

\footnotetext{
${ }^{6}$ Another set of formulas was reported in 9] but only for $D(a)$.
} 


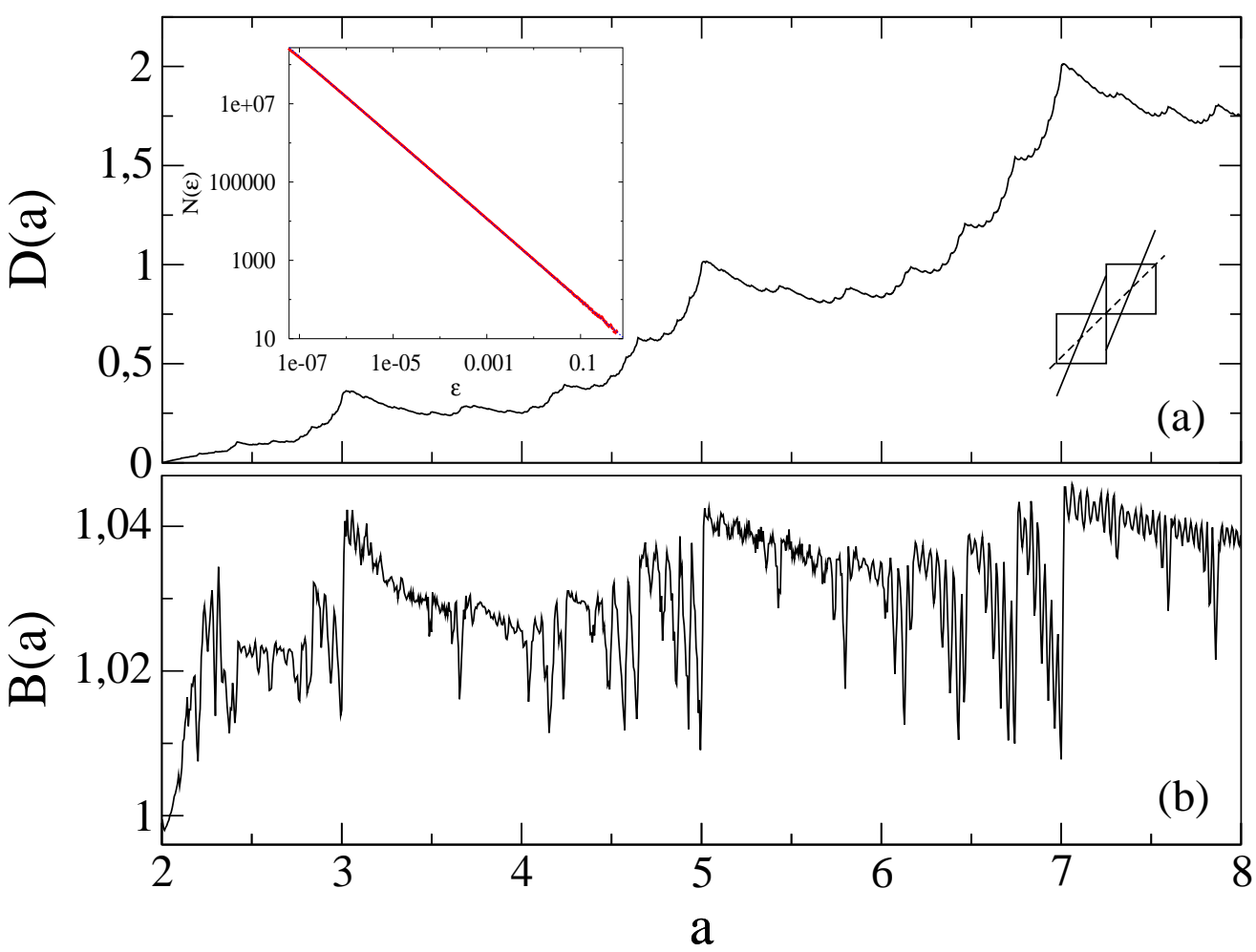

Figure 1: (a) Diffusion coefficient $D(a)$ on the interval $2 \leq a \leq 8$ with 2000 data points for the one-dimensional map (34) sketched in the lower right edge with bias $b=0$ and $a$ as the slope of the map. The inset of (a) shows standard box counting based on $10^{9}$ data points for $D(a)$, where $N$ is the number of boxes of side length $\epsilon$. The dashed line in the inset depicts the power law (51) with box dimension $B=1.039$ as computed in [30. (b) displays the box dimension $B(a)$ computed locally on a regular grid of subintervals of size $\Delta a=0.006$ centered around $a$. For each subinterval a data set of $10^{6}$ values has been used, and a running average was performed over any three neighboring $B(a)$. Both figures, except the inset, are from [30.

\subsection{Box counting for the diffusion coefficient}

Motivated by Proposition 2 and by 35, the numerical results of 30 are now reevaluated and supplemented by new, further numerical analysis. We start with the diffusion coefficient $D(a)$. Corollary 2 states that $B(a)=1$ for all intervals $\Delta a$, which is at variance with the results presented in Fig. (1. However, in contrast to the standard box counting assumption (51), Proposition 2 is compatible with the existence of multiplicative logarithmic terms by giving upper bounds for their exponents. The discussion in Subsection 4.2 shows that these terms do indeed exist.

In detail, Corollary 2 states an upper bound for the box counting function $N(\epsilon)$ of $D(a)$ of

$$
N(\epsilon) \leq K_{4} \epsilon^{-1}(1-\ln \epsilon)^{2} .
$$

This motivates us to plot the product $N \epsilon$ as a function of $-\ln \epsilon$ : For small enough $\epsilon$ and in double-logarithmic representation one should then see a straight line with the slope yielding the exponent of the logarithmic term. Fig. 2 numerically verifies the existence of this term for $D(a)$ on $2 \leq a \leq 8$ : There clearly exists a non-zero exponent, however, in the numerics $-\ln \epsilon$ is not large enough to overcome the additive constant in (52) for producing a straight line.

In Fig. 2 three data sets have been plotted consisting of different numbers of data points for $D(a)$. The bending off of the graphs at larger $-\ln \epsilon$ reflects that box counting starts to resolve the single points of all the underlying data sets: From the figure one can roughly estimate that 


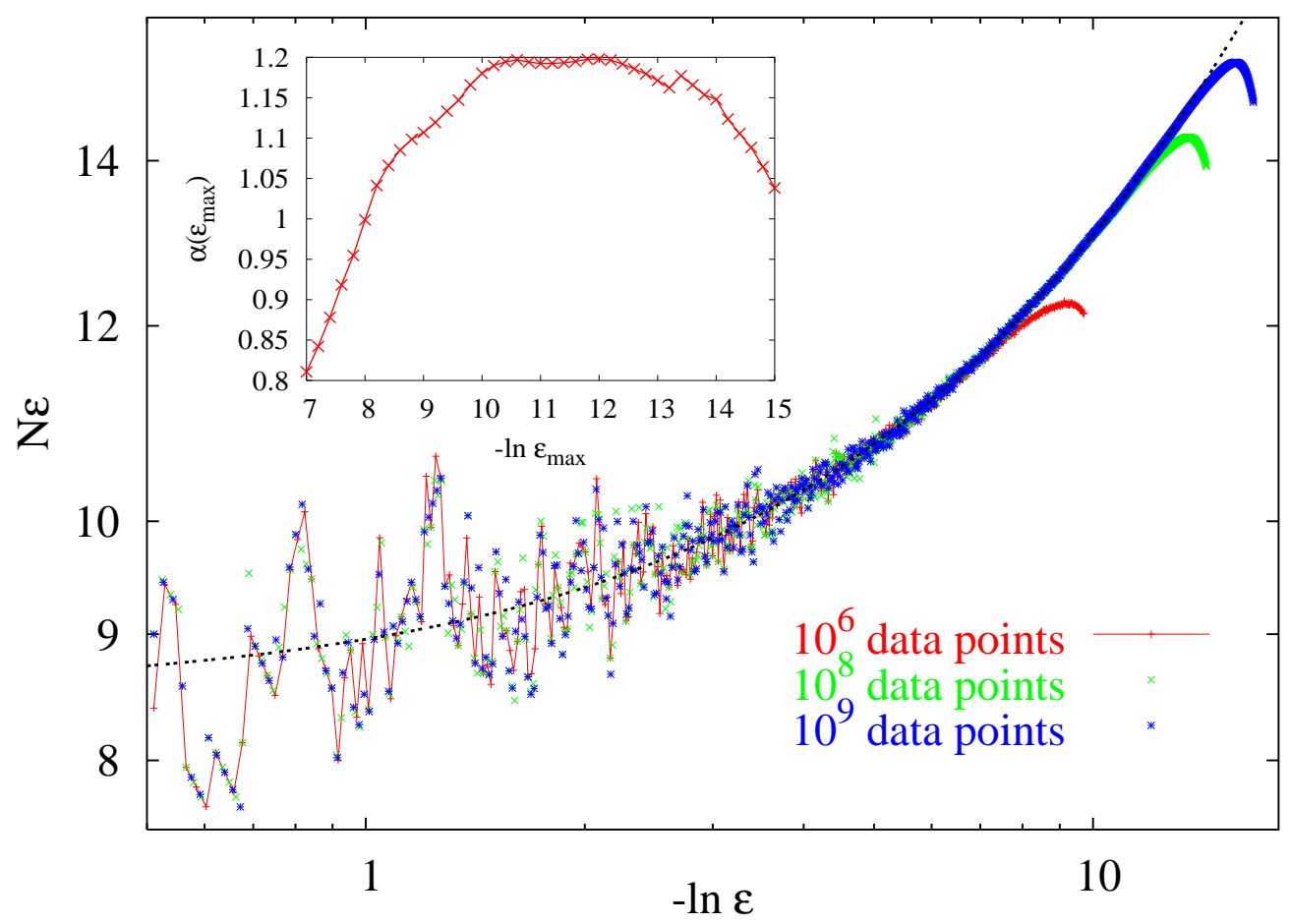

Figure 2: $N$ is the number of boxes of length $\epsilon$ needed to cover $D(a)$ shown in Fig. 11 (a) generated from $10^{6}, 10^{8}$ and $10^{9}$ data points. Motivated by (52), in contrast to the inset of Fig. 1 (a) here we plot the product $N \epsilon$ as a function of $-\ln \epsilon$ double-logarithmically. The dashed black line represents a three-parameter fit for the largest data set over $0.5 \leq-\ln \epsilon \leq 12$ with the functional form of (53). The inset shows results for the exponent of the logarithmic correction $\alpha$ obtained from fits where we vary the upper bound $\epsilon_{\max }$ of the fit interval.

for a data set of $10^{6}$ points for $D(a)$ deviations set in around $-\ln \epsilon_{\text {cut }} \simeq 7$, or $\epsilon_{\text {cut }} \simeq 10^{-3}$. Compared with a separation of $\delta a=6 \cdot 10^{-6}$ between any two data points along the $a$-axis, this yields a difference of about three orders of magnitude. The same order of magnitude argument holds if one compares $\epsilon_{\text {cut }}$ obtained approximately for $10^{8}$ data points from Fig. 2 with the corresponding separation of $\delta a=6 \cdot 10^{-8}$ between any two data points. This leads to the prediction that for the set of $10^{9}$ data points $-\ln \epsilon_{\text {cut }} \simeq 14$ in Fig. 2

Inspired by (52), we now fit the box counting results with the function

$$
N(\epsilon)=K_{5} \epsilon^{-1}\left(1+K_{6} \ln \epsilon\right)^{\alpha}
$$

instead of (51). If this fit function reproduces the numerically computed $N(\epsilon)$ reasonably well, Proposition 2 predicts that $0 \leq \alpha \leq 2$. However, we emphasize that this Proposition only gives us a strict upper bound - it does not actually tell us the "true" functional form of the whole graph. We have indeed checked that fit functions others than (53), which also obey (52), work similarly well. In order to be close to Proposition 2 we stick to the fit function (53) in the following.

The dashed black line in Fig. 2 shows a fit of the box counting results for $10^{9}$ data points of $D(a)$ with this functional form 7 The inset of Fig. 2 depicts results for the exponent $\alpha$ computed from different fit intervals $\left[0.5,-\ln \epsilon_{\max }\right]$ for the same data set of $10^{9}$ points. It indicates convergence towards $\alpha \simeq 1.2\left(-\ln \epsilon_{\max } \rightarrow 12\right)$. The decrease for $-\ln \epsilon_{\max }>12$ is well in agreement with the cutoff predicted above, which is due to the limited data set. Note

\footnotetext{
${ }^{7}$ For all fits the nonlinear least-squares Marquardt-Levenberg algorithm as implemented in gnuplot 4.0 has been used.
} 

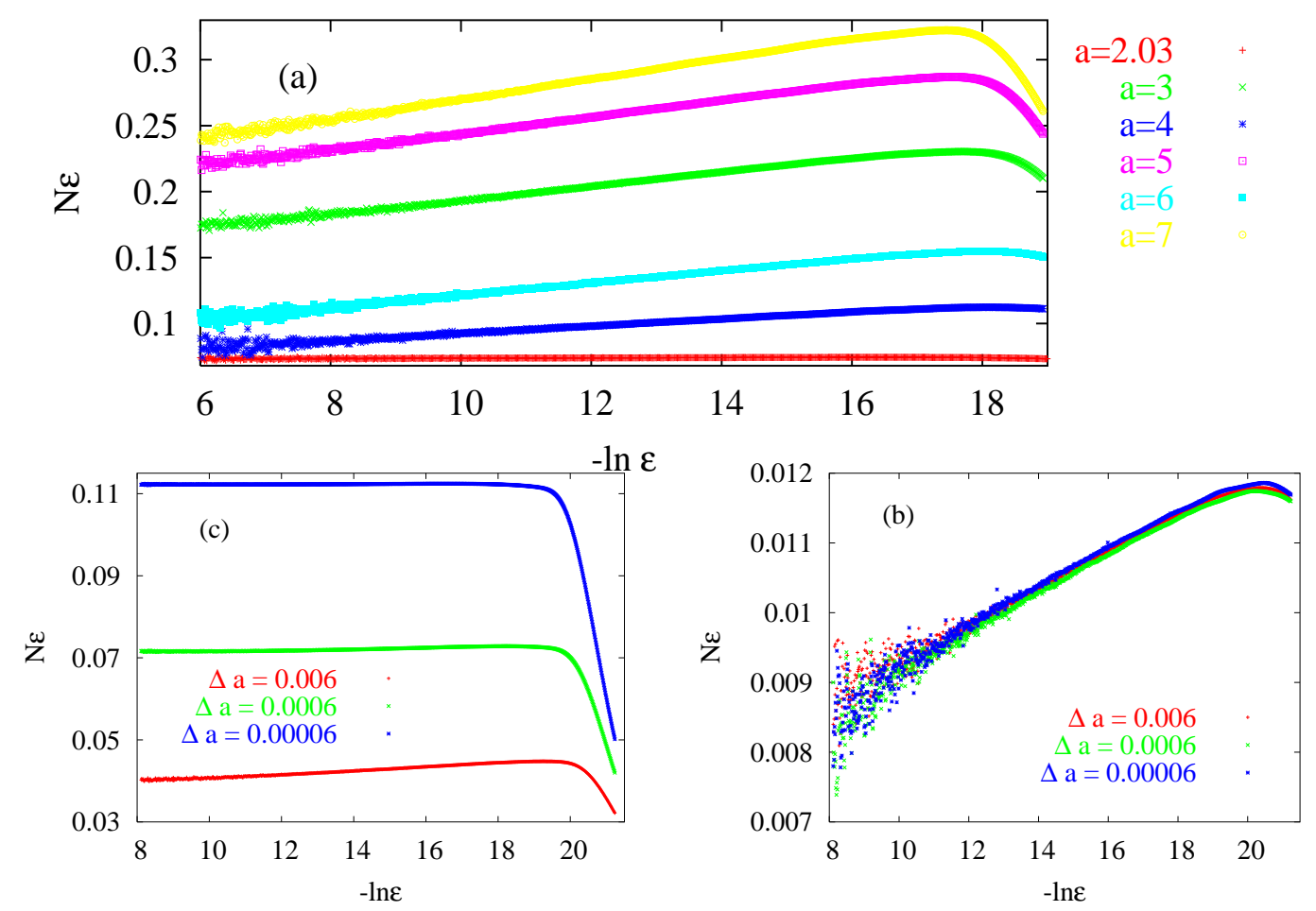

Figure 3: Local variation of the product $N \epsilon$ needed to cover $D(a)$ around integer values of $a$ : (a) shows results for parameter intervals of size $\Delta a=0.06$ centered around different $a$, based on $10^{8}$ data points. (b) displays results for subintervals $\Delta a$ all centered around $a=4$, whereas in (c) all subintervals converge towards $a=5$. In (b) and (c) the graphs have been scaled by multiplying $\epsilon$ with the order of magnitude difference between the different values for $\Delta a$.

that the cutoff sets in much later than the beginning of the plateau. Hence we conclude that for a data set of $10^{9}$ points for $D(a), 2 \leq a \leq 8$, and by assuming the fit function (53), the numerical value for the exponent of the logarithmic term is $\alpha \simeq 1.2$. This is again in agreement with Proposition $2^{8}$ Note that fits by (53) do not tell the full story: The numerically exact data in Fig. 2 show the existence of a non-trivial fine structure pointing towards more complicated functional forms for the "true" $N(\epsilon)$, which should reflect the intricate structure of $D(a)$ in Fig. 1 (a). These irregularities may not be understood as numerical errors.

After having verified the existence of logarithmic contributions on large parameter intervals we now look at local variations of the exponent $\alpha$. This is demonstrated by doing box counting for $D(a)$ on small intervals around integer values of $a$. Fig. 3 (a) reveals that there exist two families of curves: The one for even $a$ is at the bottom of this figure, whereas the one for odd $a$ is on top. Additionally, all graphs show up such that the ones for larger slopes are always on top in both groups thus creating an oscillatory structure.

We first consider the special case $a=2.03$, where according to Fig. 3 (a) $\alpha \simeq 0$. Note that $D(2)=0$, correspondingly the parameter region just above $a=2$ marks the onset of diffusion, cf. Fig. 1 (a). As described in 27, 28, 32, for $a \rightarrow 2$ there is asymptotic convergence of $D(a)$ to the simple random walk solution $D(a)=(a-2) /(2 a)$. This physical argument explains why $\alpha \rightarrow 0(a \rightarrow 2)$. There is a trend that larger even integer slopes in (a) give $0 \leq \alpha \leq 1$ whereas odd $a$ give $1<\alpha \leq 2$. Unfortunately, the fits producing these results are very unstable, hence even these rough estimates should be taken with care. In any case, the indicated order

\footnotetext{
${ }^{8}$ We have checked that these fit results do not significantly depend on the choice of the initial seeds for our three fit parameters and that the asymptotic standard error for them is less than $10 \%$ for $-\ln \epsilon_{\max }>10$. However, in our view quantitative error estimates are not reliable in this case, because we may not assume that the residua are normally distributed random variables.
} 
of magnitude of $\alpha$ appears to be in agreement with Proposition 2, Our fits furthermore suggest that not only $\alpha$ is a function of $a$ but also that the other two parameters in (53) are locally varying. This agrees with conclusions drawn in [30.

Figs. 3 (b) and (c) provide a more detailed local analysis by looking at successively smaller subintervals around two specific slopes. While (c) suggests $\alpha \rightarrow 0(\Delta a \rightarrow 0)$ around $a=5$, (b) with $a=4$ yields approximately $\alpha \rightarrow 1(\Delta a \rightarrow 0)$ Note that the graphs in (b) and (c) have been scaled as described in the figure. Interestingly, this transformation leads to a collapse onto a master curve in (b), whereas it does not work that way in (c). Similar observations have been reported in 35]. Together with the analytical results of Subsection 4.2, Fig. 3 thus demonstrates remarkable continuity properties of $D(a, b)$ around integer slopes, which strongly depend on the direction in parameter space.

These differences between graphs for odd and even $a$ are consistent with the local box counting dimension displayed in Fig. 1 (b): They suggest that the oscillatory structure in $B(a)$ actually reflects local variations of the parameters in (53) determining the logarithmic corrections, erroneously being fit in [30] with the standard box counting equation (51) instead of taking the existence of logarithmic terms into account. This result is confirmed by covering small parameter regions around $a=4$ and $a=5$ with non-overlapping sequences of subintervals and looking for local variations of the box counting results. Again, one finds oscillations that roughly correspond to the ones in Fig. 1(b). Although there is no linear functional relationship between $\alpha(a)$ and $B(a)$, one may thus argue that Fig. 1 (b) tells us something about the magnitude of local logarithmic corrections.

We remark that with the computing power available to us it was impossible to produce a graph like Fig. 1(b) for local values of $\alpha$, because for each $\alpha(a)$ box counting would have required a data set of at least $10^{9}$ values of $D(a)$. Such large data sets appear to be necessary because of monotonicity of the exponents: if $E$ is a subset of $F$ then $\alpha(E) \leq \alpha(F)$. Local variations of $\alpha$ thus pose a serious problem to any numerical box counting analysis, since eventually $\alpha(a)$ should always converge to the largest local exponent. However, if this exponent is exhibited just on a tiny subinterval it could be extremely tedious to detect it numerically. This argument of course also applies to our previous result of $\alpha \simeq 1.2$ for $D(a)$ on $2 \leq a \leq 8$, which strictly speaking only holds for the given data set of $10^{9}$ points. We cannot exclude that some tiny interval of $D(a)$ eventually yields a larger value of $\alpha$. In other words, the goal of our numerical analysis cannot be to compute unambiguous values for any exponents but rather to demonstrate qualitative and quantitative order-of-magnitude agreement with Proposition 2 .

\subsection{Box counting for the drift}

We continue our numerical analysis by investigating the parameter dependence of the drift, or current, $J(a, b)$. As for the diffusion coefficient, we start with a brief reminder of previous results in form of Fig. 4. Like Fig. 1, it displays a highly oscillatory structure both in the drift as well as in the local box dimension as functions of $a$ for fixed $b$, where $B(a)$ has been computed according to (51) by again disregarding any logarithmic corrections. Note particularly the pronounced minima at odd integer values. As before, we now reevaluate these findings on the basis of Proposition 2 by taking logarithmic terms into account.

Fig. 5 numerically confirms the existence of logarithmic corrections for $J(a, b)$ : There exist non-zero exponents $\alpha$ as allowed by Proposition 2, Note particularly the pronounced, different fine structures of both curves displayed in the main part, which are much stronger than in Fig. 2 for $D(a)$. Due to these oscillations, in case of $J(a, b)$ it is numerically very difficult to extract reliable values for the exponents $\alpha$ by using (153). The two fits included in the main graph yield an order of magnitude of $\alpha \simeq 0.1$, which matches to Proposition 2 .

The inset of Fig. 5 is analogous to Fig. 3 (a) in that it shows box counting results for the current $J(a, b), b=0.01$, mostly at integer values of the slope $a$. Note that $J(2,0.01) \simeq 0$ [15], which marks the onset of the drift. As we have argued for the diffusion coefficient, at $a=2.03$ we are thus in a random walk regime for which one may expect $\alpha \simeq 0$, as is shown in the figure.

${ }^{9}$ Again, the fit results are highly unstable, so the latter value should be taken with care. 

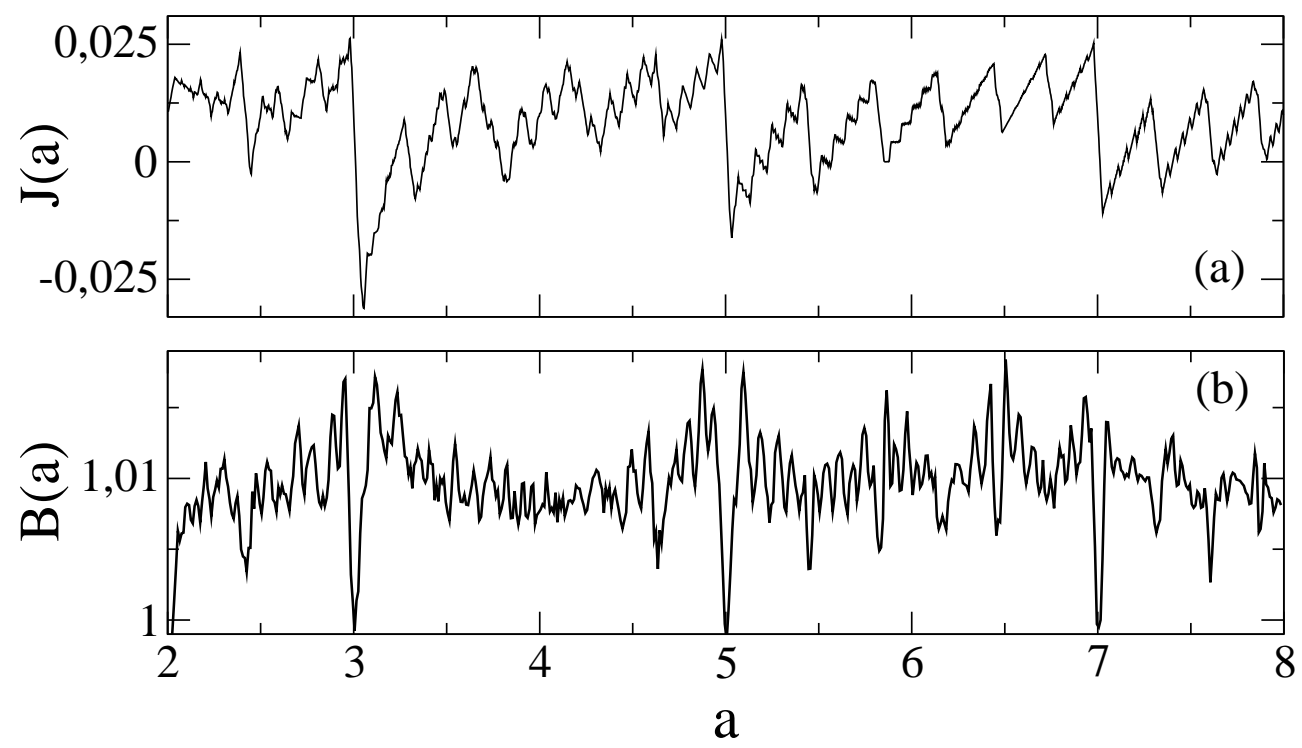

Figure 4: (a) Drift $J(a)=J(a, 0.01)$ on the interval $2 \leq a \leq 8$ based on 2000 data points. As in Fig. 1 (b) depicts the box dimension $B(a)$ computed locally on a regular grid of subintervals of size $\Delta a=0.01$ averaged over any three neighboring points. Both figures are from [30].

However, $\alpha(a) \neq 0$ in all the other cases of the inset suggesting again a local variability of $\alpha$ for $J(a, b)$, at least around integer values of $a$. As in Fig. 3 (a) there exist two family of curves, one for even $a$ at the bottom and one for odd $a$ on top of the figure. There is also again an additional ordering, however, here it is such that curves for larger slopes are always at the bottom in both families of graphs, except at $a=2.03$. The additional graph for $a=7.09$ exemplifies the strong local variability of $\alpha$ around $a=7$ which, as well as the difference between odd and even slopes for box counting results of the drift, agrees with the oscillations in the local box dimension $B(a)$ shown in Fig. 4

Fits for all the inset curves yield a trend towards small exponents around even and somewhat larger values around odd slopes with an order of magnitude of $0 \leq \alpha \leq 1$, which appears to be consistent with Proposition 2. However, we emphasize again that these results give only a rough indication for the numerical reasons discussed above. Exact results are only available for special cases: As we have discussed in Subsection 4.2, $J(a, b)=b$ for constant $a \in \mathbb{N}$ under variation of $b$, where we thus have $\alpha=0$, linear response and a caricature of Ohm's law. For general $a$ one finds that $J(a, b) /(b|\log | b||)$ is bounded but has no limit for $b \rightarrow 0$ [15] pointing towards logarithmic corrections.

We have also qualitatively checked graphs of $D(a, b)$ and $J(a, b)$ for other parameter values, that is, by choosing different values for $a$ and $b$ fixed in the parameter plane and studying the resulting functions of the remaining free control parameters. Qualitatively, we obtain results that are analogous to the ones discussed above.

\subsection{Continuity properties of the diffusion coefficient at integer slopes}

The previous two subsections demonstrated a very peculiar behaviour of local box counting results for drift and diffusion coefficient around integer slopes $a$ at fixed values of the bias $b$. Subsection 4.2, in turn, gave exact analytical expressions for the difference $D_{a}\left(b^{\prime}\right)-D_{a}(b)$ of the diffusion coefficient as a function of $\Delta b=b^{\prime}-b$ at integer $a$ in the limit of small $\Delta b$. This suggests to numerically study the continuity properties of $D_{a}(b)$ at fixed integer values of $a$ in more detail.

In order to access suitably small values of the parameter $\Delta b$, we have employed the Fortran90 library mpfun90 [1] for arbitrary-precision arithmetic. Using this library we have calculated the 


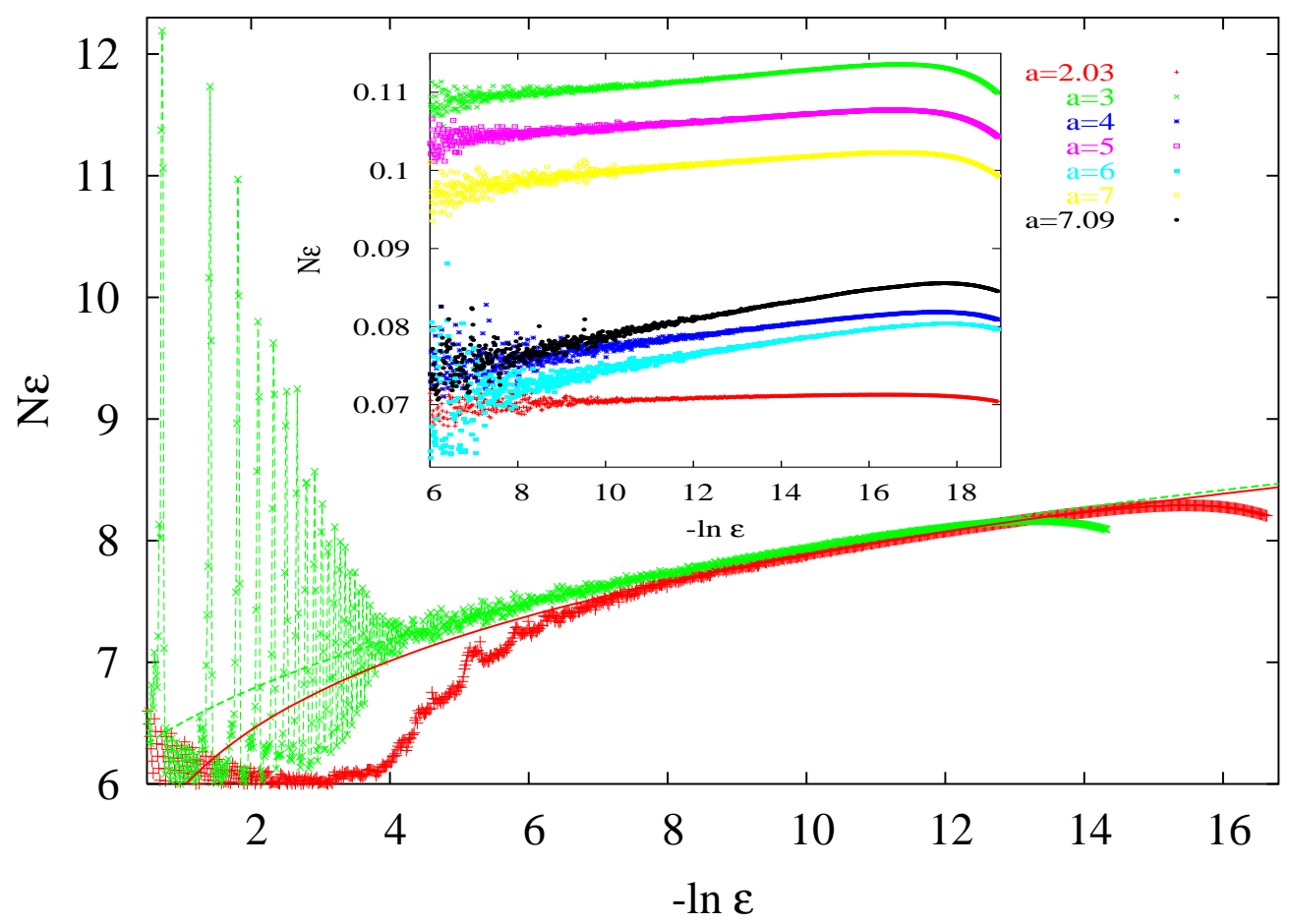

Figure 5: Main graph: Product $N \epsilon$ as a function of $-\ln \epsilon$ for the $\operatorname{drift} J(a, b)$ over the interval $2 \leq a \leq 8$ at $b=0.01$ (red ' + ' symbols, based on $10^{9}$ data points) and at $b=0.49$ (green ' $\mathrm{x}$ ' symbols, based on $10^{8}$ data points). Included are two fits over the intervals $8 \leq-\ln \epsilon \leq 13$ $(b=0.01)$ and $4 \leq-\ln \epsilon \leq 11.5(b=0.49)$. Inset: Local variation of the product $N \epsilon$ as a function of $-\ln \epsilon$ for parameter intervals of size $\Delta a=0.06$, mostly centered around integer values of $a$ and based on $10^{8}$ data points. The graph for $a=7.09$ demonstrates that, in agreement with Fig. 4 (b), there exist strong local fluctuations of the box counting functions under variation of the slope $a$ of the map.

difference quotient $\left(D_{a}\left(b^{\prime}\right)-D_{a}(b)\right) /\left(b^{\prime}-b\right)$ of $D$ with fixed $b$ at values of $\Delta b$ down to $10^{-200}$. Figure 6 (a) shows a subset of our results for $a=3$ and $a=4$ at fixed $b \in\{-0.5,0\}$. There is excellent agreement between the numerical results and the analytical observations (11) and (2) of Subsection 4.2 predicting straight lines. This agreement is as good to the limits of attainable precision, and has been checked for other integer values than those shown in Fig. 6.

Figure 6(b) depicts the diffusion coefficient $D_{a}(b)$ at $a=4$ and a blowup around $b=0$, which corresponds to the two curves in (a) at this $a$ value. Note that there is reflection symmetry for $D_{a}(b)$ with respect to $b=-0.5$ and $b=0$. One can see that at $b=-0.5$, where the difference quotient in (a) displays a multiplicative logarithmic term, $D_{a}(b)$ in (b) exhibits a global maximum in form of a sharp cusp. The global minimum at $b=0$, on the other hand, is approached in a rather smooth, oscillatory manner yielding a rounded-off shape, see the inset in (b). This relates to the difference quotient curve in (a) with zero logarithmic term. Analogous observations are made for $a=3$, where $D_{a}(b)$ exhibits local maxima both at $b=-0.5$ and at $b=0$, and for other integer slopes. We remark that the quite regular structure of $D_{a}(b)$ in $(\mathrm{b})$, particularly around both local extrema, resembles very much the one of the fractal generalized Takagi functions studied in [27, 14, 32].

Observation (3) generalizes observations (11) and (2) by stating that logarithmic corrections are typical for parameter values of $b$ yielding Markov partitions. In [26, 27, 29, 15, it has been shown (for $b=0$ ) that Markov partition parameter values identify local maxima and minima of the parameter-dependent diffusion coefficient by relating them to ballistic and localized orbits 

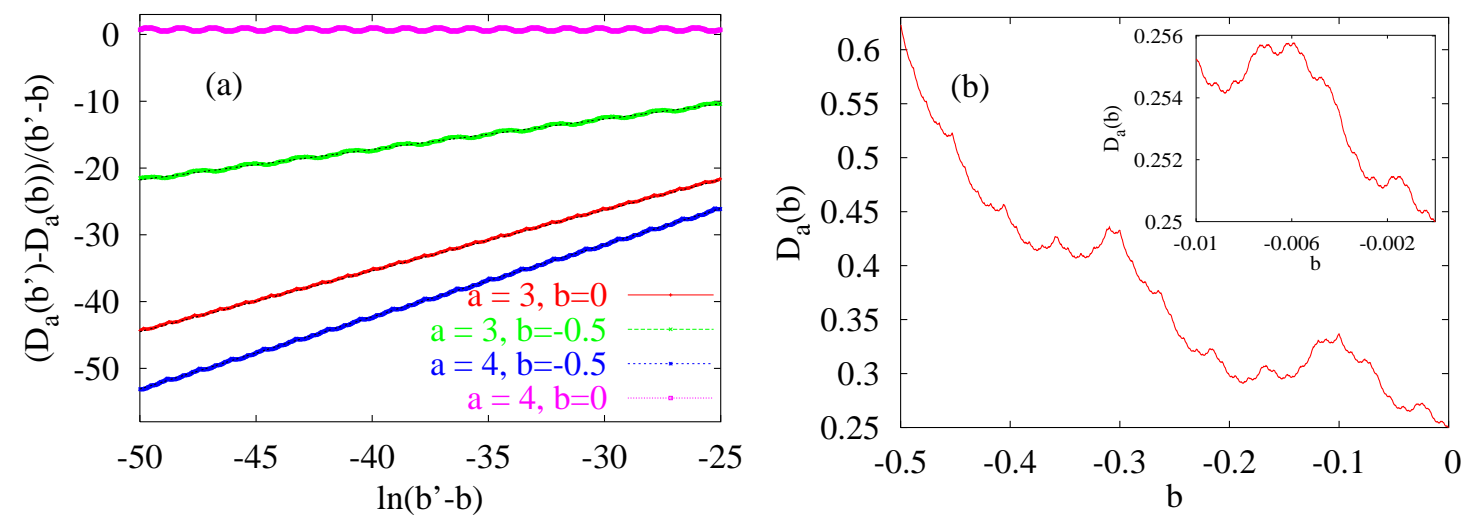

Figure 6: (a) Difference quotient $\left(D_{a}\left(b^{\prime}\right)-D(b)\right) /\left(b^{\prime}-b\right)$ as a function of $\ln \left(b^{\prime}-b\right)$ at integer values of $a \in\{3,4\}$ and with fixed $b \in\{-0.5,0\}$. Each curve is based on $10^{5}$ data points. Included are best fit curves (black dashed lines) whose fitted slopes (from bottom to top: $3 /(2 \ln 4), 1 / \ln 3$ and $1 /(2 \ln 3))$ agree with the analytic predictions of Subsection 4.2 to four significant figures. The case $a=4, b=0$ clearly has slope zero, as predicted. The barely visible fine scale oscillations of each curve reflect higher order correlations in these quantities. (b) Diffusion coefficient $D_{a}(b)$ at $a=4$ for $-0.5 \leq b \leq 0$ and a magnification of the region around $b=0$. For each curve 2000 data points have been computed from exact analytical solutions for $D_{a}(b)$ [15]. These curves form the basis for the two graphs at $a=4$ displayed in (a).

of the critical points of the lifted map, respectively. One may thus speculate that the above numerical observation holds true for local extrema on finer scales, that is, that local cusps in $D_{a}(b)$ reflect logarithmic corrections in the local difference quotient, whereas rounded-off local extrema signify the lack of logarithmic terms. See also [35] for related results. Furthermore, in Fig. 6 (a) we deliberately restricted the range of $\ln \left(b^{\prime}-b\right)$ so that, upon very close scrutiny, a fine structure of all curves can be seen on top of the straight line behaviour. Fig. 6 (b) suggests that this oscillatory fine structure, which yields higher order corrections to the analytical results of Subsection 4.2, is induced by the fine structure of $D_{a}(b)$.

We have also numerically investigated the accuracy of observation (4) in Subsection 4.2 . Its main statement is that at fixed $\Delta b$ and with $b$ values taken uniformly from the interval $[0,1 / 2)$, the quantity $\left(D\left(b^{\prime}\right)-D(b)\right) / \Delta b \sqrt{-\ln \Delta b}$ should be distributed like a mixture of centered Gaussians, that distribution being independent of the particular value of $\Delta b$. In fact what is typically seen at integer slopes is a distribution rather close to a pure Gaussian. We have tested this using the technique of quantile-quantile plotting (qqplots) as well the standard ShapiroWilk normality test. Both tools were implemented in the statistical package $\mathrm{R}[42$.

Figure 7 presents results obtained for three sets of data with the slope fixed at $a=4$. For larger $a$, the results become closer to a fixed Gaussian, as the function $g(x)$ in (46) becomes more dominated by the $x$ term which has no $b$ dependence. Here however, deviations from Gaussianity can be seen, at least for sufficiently small $\Delta b$. In the three parts of Figure 7 the red line with slope $\sigma$ and zero offset $\mu$ shows the theoretical result for a Gaussian distribution with standard deviation $\sigma$ and mean $\mu$, with those parameters here taken as those of our data set. As can be seen, all our distributions show close agreement with this curve. However, the Shapiro-Wilk normality test is more discerning: in (a) $\Delta b=10^{-10}$ and we obtain a p-value of only 0.008 , well below the significance level for rejecting the null hypothesis of normality. In (b) $\Delta b=10^{-50}$ and we get a p-value of 0.25 , demonstrating that this distribution is indeed very close to a pure Gaussian. It is however likely that the deviations from Gaussianity in (a) are rather due to deterministic effects arising from the relatively large value of $\Delta b$ chosen and not from the nature of the true limiting distribution being a mixture of Gaussians predicted by observation (4). It seems that the dominant behaviour when the distribution seems to have 


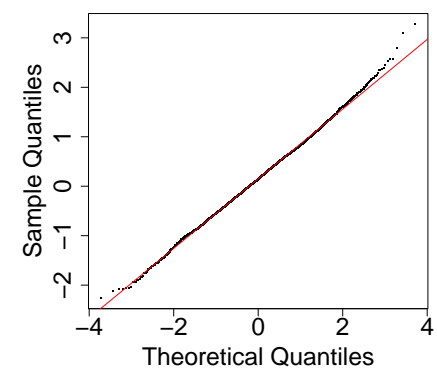

(a)

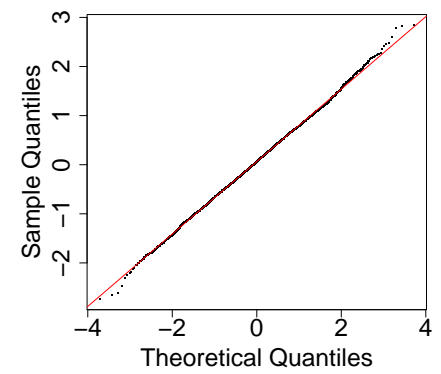

(b)

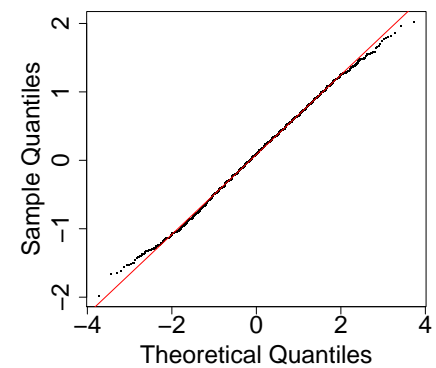

(c)

Figure 7: (a) Normal quantile-quantile plot at $a=4$ for the distribution of $D\left(b^{\prime}\right)-D(b)$, scaled by $\Delta b \sqrt{-\ln \Delta b}$, with chosen $\Delta b=b^{\prime}-b=10^{-10}$ held constant and $b$ picked from a uniform distribution on $[0,1 / 2)$. The red line with slope $\sigma$ and zero offset $\mu$ would be the result for a Gaussian distribution with standard deviation $\sigma$ and mean $\mu$. Here the numerically obtained values of these parameters were used for the fit. (b) As (a) but with $\Delta b=10^{-50}$. (c) As (b) but with the range of $b$ restricted to $[0,0.005)$.

converged is not detectably different from a pure Gaussian. We note that despite this, the two distributions in (a) and (b) are similar and both have mean close to zero, demonstrating that we have no disagreement with observation (4), merely that its details are too sensitive to check numerically.

It is however possible to go further numerically, for example one can also study the nature of the distribution obtained when $b$ is taken from a subinterval of $[0,1 / 2)$, which as can be seen in Fig. 7 (c) leads in the case of integer $a$ to distributions with rather more fine structure than the nice curves seen in Figs. 7 (a) and (b). This is clear evidence of the deterministic nature of the underlying system in the form of strong correlations at fine scales. In this case the Shapiro-Wilk p-value is about 0.001 .

As far as observation (4) is concerned, away from integer values of $a$ quite different behaviour is seen thus clarifying that this observation is rather to be considered atypical. Here the distribution of $D$-differences is centred around zero still, but with a more sharply peaked and heavily tailed distribution than a Gaussian. These deviations persist even very close to the integer cases (e.g. at $a=3+10^{-50}$ ), though Gaussian behaviour does appear to be approached slowly in the limit of integer values.

These numerical methods can also be used to investigate variation of the continuity of the transport coefficients as $b$ is held fixed and $a$ varies, as considered in [35] and already looked at using box counting in Fig. 3. Here the maximal exponent of logarithmic correction, i.e. $D\left(a^{\prime}\right)-D(a) \sim\left|a^{\prime}-a\right|\left(\ln \left|a^{\prime}-a\right|\right)^{2}$, can be seen for odd $a$, and though this might appear to be in contradiction to the third part of Fig. 3 for $a=5$, in fact arbitrarily close to $a=5$ the exponent tends locally to zero. Thus the box counting only sees the "typical" local behaviour and the current method is more suited for picking out atypical behaviour at specific points.

\section{Conclusions and outlook}

(1) We proved rigorously that the diffusion coefficient of deterministic random walks generated by piecewise expanding interval maps depends continuously on the maps. More precisely, for "natural" parametrizations of the maps by some parameter $\lambda$, the diffusion coefficient as a function of the parameters has a modulus of continuity not worse than $|\delta \lambda|(\log |\delta \lambda|)^{2}$. Even if all maps in the family are topologically conjugate, the detailed analysis of section 4.2 shows that the modulus of continuity cannot be expected to be better than $|\delta \lambda \cdot \log | \delta \lambda||$. This is in sharp contrast to the situation for the drift (or other averages of observables) 
that depend differentiably on parameters in this case [4. One might thus conjecture: If the maps are all topologically conjugate as in the case of integer slopes, then $\mathrm{J}$ is Lipschitz and $\mathrm{D}$ has simple logarithmic corrections. Otherwise $\mathrm{J}$ has simple logarithmic corrections and D has quadratic ones.

(2) We verified numerically the existence of logarithmic corrections in the box counting data for both the parameter dependent drift and diffusion coefficients. The computed values for the exponents of these logarithmic terms are compatible with the bounds predicted by our mathematical theory. However, we emphasize again the serious difficulties to obtain quantitatively reliable numerical results, which required to analyze huge data sets. These difficulties are due to strong local variations of these exponents and of the other control parameters governing the logarithmic corrections, as we find numerically.

These new numerical results correct and amend the previous box counting analysis of Klages and Klauß [30] along the lines conjectured by Koza [35. Our model thus generates interesting examples of fractals for which the definition of the standard box counting dimension is misleading. We conclude that the (local) non-integer variations of the box dimension reported in [30] actually reflect non-trivial local variations of the parameters of the logarithmic corrections.

We have furthermore numerically verified analytical predictions for the difference quotient of the diffusion coefficient as a function of the bias at integer slopes. These results suggest that the existence of logarithmic corrections is intimately related to the shape of the extrema in the diffusion coefficient curves.

(3) In 33 a nonlinear generalization of our present model has been studied, which exhibits anomalous diffusion generated by marginal fixed points. Computer simulations led to conjecture that the anomalous diffusion coefficient of this map is discontinuous on a dense set of parameter values. It would be interesting to check this conjecture mathematically.

These fractal transport coefficients also seem to provide a nice testing ground for methods of multifractal analysis [11.

Another important problem is to check whether such logarithmic corrections in transport coefficients might also be expected to occur in more 'physical' systems, which are perhaps even accessible experimentally. This seems to be strongly related to the question whether a family of physical dynamical systems shares the same topological conjugacy class under parameter variation.

\section{Acknowledgements:}

G.K. and R.K. thank C. Beck, C. Dettmann and M. Pollicott, the organizers of the LMS Durham Symposium on Dynamical Systems and Statistical Mechanics in July 2006, where this work was started, for their kind invitation. R.K. and P.J.H. were supported by a grant from the British EPSRC under EP/E00492X/1.

\section{References}

[1] D. Bailey, A Fortran-90 Based Multiprecision System, ACM Transactions on Mathematical Software 21 (1995), 379-387

[2] V. Baladi: Positive Transfer Operators and Decay of Correlations (Advanced Series in Nonlinear Dynamics, Vol 16, World Scientific, Singapore, 2000).

[3] V. Baladi, On the susceptibility function of piecewise expanding interval maps, Commun. Math. Phys. 275 (2007), 839-859.

[4] V. Baladi, D. Smania, Linear response formula for piecewise expanding unimodal maps, Preprint arxiv.org (2007). 
[5] V. Barnett Probability Plotting Methods and Order Statistics, Appl. Stat. 24 (1975), 95-108

[6] M.L. Blank, Singular effects in chaotic dynamical systems, Russian Acad. Sci. Dokl. Math. 47, 1-5 (1993).

[7] M. Blank, G. Keller, Stochastic stability versus localization in chaotic dynamical systems, Nonlinearity 10, 81-107 (1997).

[8] O. Butterley, C. Liverani, Smooth Anosov flows: correlation spectra and stability, J. Modern Dynamics 1 (2007) 301-322.

[9] G. Cristadoro, Fractal diffusion coefficient from dynamical zeta functions, J. Phys. A: Math. Gen. 39 (2006) L151-L157

[10] D. Dolgopyat, On differentiability of SRB states for partially hyperbolic systems, Invent. Math. 155 (2004) 389-449.

[11] A.Faccini, S.Wimberger, A.Tomadin, Multifractal fluctuations in the survival probability of an open quantum system, Physica A 376 (2007) 266-274.

[12] L. Flatto, J.C. Lagarias, The lap-counting function for linear mod one transformations II: the Markov chain for generalized lap numbers, Ergod. Th.\& Dynam. Sys. 17 (1997), 123-146.

[13] L. Flatto, J.C. Lagarias, The lap-counting function for linear mod one transformations III: the period of a Markov chain, Ergod. Th.\& Dynam. Sys. 17 (1997), 369-403.

[14] P. Gaspard, R. Klages, Chaotic and fractal properties of deterministic diffusion-reaction processes, Chaos 8 (1998) 409-423.

[15] J. Groeneveld, R. Klages, Negative and nonlinear response in an exacly solved dynamical model of particle transport, J. Stat. Phs. 109 (2002), 821-861.

[16] F. Hofbauer, Maximal measures for simple piecewise monotonic transformations, Z. Wahrscheinlichkeitstheorie verw. Geb. (now: Probab. Th. Rel. Fields) 52 (1980), 289-300.

[17] F. Hofbauer, The maximal measure for linear mod one transformations, J. London Math. Soc. (2) 23 (1981), 92-112.

[18] F. Hofbauer, G. Keller, Ergodic properties of invariant measures for piecewise monotonic transformations, Math. Zeitschrift 180 (1982), 119-140.

[19] M. Jiang R. de la Llave, Linear response function for coupled hyperbolic attractors, Commun. Math. Phys. 261 (2006) 379-404.

[20] Y. Jiang, D. Ruelle, Analyticity of the susceptibility function for unimodal markovian maps of the interval, Nonlinearity 18 (2005) 2447-2453.

[21] G. Keller, Un théorème de la limite centrale pour une classe de transformations monotones par morceaux, C. R. Acad. Sci. Paris, Série A, 291 (1980), 155-158

[22] G. Keller, Stochastic stability in some chaotic dynamical systems, Monatshefte Math. 94 (1982), 313-333.

[23] G. Keller, Interval maps with strictly contracting Perron-Frobenius operators, Int. J. Bifurc. Chaos 9 (1999), 1777-1784.

[24] G. Keller, C. Liverani, Stability of the spectrum for transfer operators, Ann. Mat. Sc. Norm. Pisa 28 (1999), 141-152. 
[25] G. Keller, C. Liverani, A spectral gap for a one-dimensional lattice of coupled piecewise expanding interval maps, in: Dynamics of Coupled Map Lattices and of Related Spatially Extended Systems (Eds.: J.-R. Chazottes, B. Fernandez), Lecture Notes in Physics 671 (2005), pp. 115-151, Springer Verlag.

[26] R. Klages, J.R. Dorfman, Simple maps with fractal diffusion coefficients, Phys. Rev. Lett. 74 (1995) 387-390.

[27] R. Klages, Deterministic diffusion in one-dimensional chaotic dynamical systems (Wissenschaft \& Technik-Verlag, Berlin, 1996).

[28] R. Klages, J.R. Dorfman, Dynamical crossover in deterministic diffusion, Phys. Rev. E 55 (1997) R1247-R1250.

[29] R. Klages, J.R. Dorfman, Simple deterministic dynamical systems with fractal diffusion coefficients, Phys. Rev. E 59 (1999) 5361-5383.

[30] R. Klages, T. Klauß, Fractal fractal dimensions of deterministic transport coefficients, J. Phys. A: Math. Gen. 36 (2003) 5747-5764.

[31] R. Klages, I.F. Barna, L. Mátyás, Spiral modes in the diffusion of a single granular particle on a vibrating surface, Phys. Lett. A 333 (2004) 79-84.

[32] R. Klages, Microscopic chaos, fractals and transport in nonequilibrium statistical mechanics (Advanced Series in Nonlinear Dynamics, Vol 24, World Scientific, Singapore, 2007).

[33] N. Korabel, R. Klages, A.V. Chechkin, I.M. Sokolov, V.Yu. Gonchar, Fractal properties of anomalous diffusion in intermittent maps, Phys. Rev. E 75 (2007) 036213.

[34] R.Klages, unpublished.

[35] Z. Koza, Fractal dimension of transport coefficients in a deterministic dynamical system, J. Phys. A: Math. Gen. 37 (2004) 10859-10877.

[36] A. Lasota, J.A. Yorke, On the existence of invariant measures for piecewise monotonic transformations, Transactions Amer. Math. Soc. 186 (1973), 481-488.

[37] M. Mazzolena, Dinamiche espansive unidimensionali: dipendenza della misura invariante da un parametro, Master's Thesis, Roma 2 (2007).

[38] J. Rousseau-Egele, Un théorème de la limite locale pour une classe de transformations dilatantes et monotones par morceaux, Ann. Probab. 11, 772-788 (1983).

[39] D. Ruelle, Differentiation of SRB states, Commun. Math. Phys. 187 (1997), 227-241. (See also Commun. Math. Phys. 234 (2003), 185-190.)

[40] D. Ruelle, Differentiating the a.c.i.m. of an interval map with respect to $f$, Commun. Math. Phys. 258 (2005) 445-453.

[41] C. Tricot, Curves and fractal dimension (Springer, Berlin, 1995)

[42] R Development Core Team, R: A Language and Environment for Statistical Computing (R Foundation for Statistical Computing, Vienna, Austria, 2007) 